Prepared For SUbMission to JHEP

\title{
SOFTSUSY3.3.3: a program for calculating supersymmetric spectra
}

\author{
B.C. Allanach ${ }^{a}$ \\ ${ }^{a}$ DAMTP, CMS, University of Cambridge, Wilberforce road, Cambridge, CB3 0WA, United Kingdom \\ E-mail: B.C.Allanach@damtp.cam.ac.uk
}

\begin{abstract}
SOFTSUSY is a program which accurately calculates the spectrum of superparticles in the CP-conserving Minimal Supersymmetric Standard Model (MSSM), with a full flavour mixing structure. The program solves the renormalisation group equations with theoretical constraints on soft supersymmetry breaking terms provided by the user. Weak-scale gauge coupling and fermion mass data (including one-loop finite MSSM corrections) are used as a boundary condition, as well as successful radiative electroweak symmetry breaking. The program can also calculate a measure of fine-tuning. The program structure has been designed to easily generalise to extensions of the MSSM. This article serves as a self-contained guide to prospective users, and indicates the conventions and approximations used.
\end{abstract}

KEYWORDS: sparticle, MSSM 


\section{Contents}

1 Introduction $\quad 2$

1.1 The Nature of the Physical Problem 2

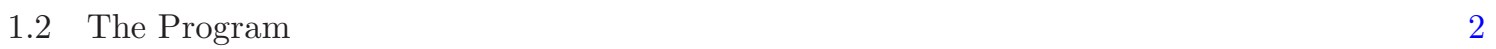

$\begin{array}{ll}1.3 \text { Aims and Layout } & 3\end{array}$

2 MSSM Parameters $\quad 3$

2.1 Supersymmetric Parameters 3

2.2 SUSY Breaking Parameters 4

2.3 Tree-Level Masses 4

3 Calculation $\quad 6$

$\begin{array}{lll}3.1 & \text { Below } M_{Z} & 6\end{array}$

$\begin{array}{lll}3.2 & \text { Initial Estimate } & 6\end{array}$

$\begin{array}{ll}3.3 \text { Gauge and Yukawa Couplings } & 7\end{array}$

3.4
MSSM Renormalisation

$\begin{array}{lll}3.5 & \text { Electroweak Symmetry Breaking } & 9\end{array}$

\begin{tabular}{ll}
3.6 & MSSM Spectrum \\
\hline
\end{tabular}

$\begin{array}{llr}3.7 & \text { Fine Tuning } & 11\end{array}$

A Running SOFTSUSY 12

A.1 Input file 12

$\begin{array}{lr}\text { B Sample Program } & 14\end{array}$

C Sample Output $\quad 16$

$\begin{array}{ll}\text { C.1 Problem flags } & 17\end{array}$

$\begin{array}{ll}\text { D Switches and Constants } & 18\end{array}$

E Object Structure 19

$\begin{array}{ll}\text { E.1 Linear Algebra } & 19\end{array}$

$\begin{array}{llr}\text { E.2 General Structure } & 19\end{array}$

$\begin{array}{llr}\text { E.3 RGE Class } & 20\end{array}$

$\begin{array}{ll}\text { E.4 QedQcd Class } & 21\end{array}$

\begin{tabular}{ll} 
E.5 & MssmSusy Class \\
\hline
\end{tabular}

E.6 SoftParsMssm Class 22

$\begin{array}{lll}\text { E.7 } & \text { MssmSoftsusy Class } & 23\end{array}$

$\begin{array}{ll}\text { E.8 MssmSoftsusyAltEwsb Class } & 27\end{array}$

$\begin{array}{lll}\text { E.9 FlavourMssmSoftsusy Class } & 27\end{array}$ 


\section{Introduction}

The Minimal Supersymmetric Standard Model (MSSM) provides an attractive weak-scale extension to the Standard Model. As well as solving the gauge hierarchy problem, it can be motivated by more fundamental models such as various string theories or supersymmetric grand unified theories. The MSSM provides a rich and complicated phenomenology. It predicts many states extra to the Standard Model (sparticles) and their indirect empirical effects and direct detection are vital for verification of the MSSM. Models that are more fundamental than the MSSM can provide stringent constraints upon the way supersymmetry (SUSY) is broken, with important implications for the spectrum which in turn affects the signatures available in experiments. It is therefore desirable to construct a calculational tool which may provide a spectrum and couplings of the MSSM sparticles so that studies of the capabilities of colliders, extraction of high scale parameters (if a signal is observed) and studies of constraints on the models are enabled. We present such a tool (SOFTSUSY) in this article.

\subsection{The Nature of the Physical Problem}

The determination of sparticle masses and couplings of SUSY particles in the R-parity conserving MSSM is the basic problem. Low energy data on Standard Model fermion masses, gauge couplings and electroweak boson masses are to be used as a constraint. SUSY radiative corrections to these inputs from sparticle loops depend upon the sparticle spectrum, and must be calculated. Theoretical constraints on the SUSY breaking parameters from an underlying theory are often imposed at a high renormalisation scale, perhaps resulting from a super gravity or string theory. Often, the theoretical constraints drastically reduce the number of free parameters in the SUSY breaking sector (which numbers over 100 in the unconstrained case). These constraints then make phenomenological analysis tractable by reducing the dimensionality of parameter space sufficiently so that parameter scans over a significant volume of parameter space are possible. Finally, the MSSM parameters must also be consistent with a minimum in the Higgs potential which leads to the observed electroweak boson masses.

This problem has been addressed many times before in the literature (see for example [1-5]), with varying degrees of accuracy in each part of the calculation. It is our purpose here to provide a tool which will solve the problem with a high accuracy, including state-of-the-art corrections. Similar problems in the context of MSSM extensions ${ }^{1}$ have also been studied. In anticipation of new forms of SUSY breaking constraints and new MSSM extensions, we designed the tool to be flexible and easily extended.

\subsection{The Program}

SOFTSUSY has been written in object-oriented $\mathrm{C}++$ but users may use an executable program with input either in the SUSY Les Houches Accord [29] format or from command-line arguments. For users wishing to call SOFTSUSY from their own programs, the user interface is designed to be C-like to aid users that are unfamiliar with object orientation. Accuracy and ease of generalisation have taken priority over running speed in the design. For example, full three family mass and Yukawa matrices may be employed, rather than the more usual dominant third family approximation. The publicly released codes ISASUGRA (which comprises part of the ISAJET package [5]) and SUSPECT [4] use the dominant third family approximation, for example. The full three-family choice slows the renormalisation group evolution significantly, but will facilitate studies of sparticle or quark mixing.

\footnotetext{
${ }^{1}$ By MSSM extension, we mean an extension applicable near the weak scale.
} 
The running time is not foreseen as a bottleneck because it is a matter of a couple of seconds on a modern PC, and will certainly be negligible compared to any Monte-Carlo simulation of sparticle production and decay in colliders. It is possible for the user to specify their own high scale boundary conditions for the soft SUSY breaking parameters without having to change the SOFTSUSY code. For the convenience of most users however, the most commonly used high-scale boundary conditions are included in the package.

The code can be freely obtained from the SOFTSUSY web-page, which currently resides at the address

http://projects.hepforge.org/softsusy/

Installation instructions and more detailed technical documentation of the code may also be found there.

SOFTSUSY is a tool whose output could be used for studies of MSSM sparticle searches [6] by using event generators such as HERWIG [7], or other more theoretical or astrophysical studies. For a review of SUSY tools on offer (which may use the output from SOFTSUSY), see Ref. [8].

\subsection{Aims and Layout}

The main aims of this article are to provide a manual for the use of SOFTSUSY, to describe the approximations employed and to detail the notation used in order to allow for user generalisation. There have been other articles published on the comparison of the calculation in SOFTSUSY with those of other codes [9], and so we decline from including such information here.

The rest of this paper proceeds as follows: the relevant MSSM parameters are presented in sec. 2. The approximations employed are noted in sec. 3, but brevity requires that they are not explicit. However, a reference is given so that the precise formulae utilised may be obtained in each case. The algorithm of the calculation is also outlined. Technical information related to running and extending the program is placed in appendices. A description of how to run the command-line interface is given in appendix A, including information on the input-file. Appendix B gives an example of a main program, useful if the user wants to call SOFTSUSY from his or her main program. The sample output from this program is displayed and explained in appendix C. The use of switches and constants is explained in appendix D. Finally, in appendix E, a description of the relevant objects and their relation to each other is presented.

\section{MSSM Parameters}

In this section, we introduce the MSSM parameters in the SOFTSUSY conventions. Translations to the actual variable names used in the source code are shown in appendix E.

\subsection{Supersymmetric Parameters}

The chiral superfields of the MSSM have the following $G_{S M}=S U(3)_{c} \times S U(2)_{L} \times U(1)_{Y}$ quantum numbers

$$
\begin{array}{ll}
L:\left(1,2,-\frac{1}{2}\right), \quad \bar{E}:(1,1,1), & Q:\left(3,2, \frac{1}{6}\right), \quad \bar{U}:\left(\overline{3}, 1,-\frac{2}{3}\right), \\
\bar{D}:\left(\overline{3}, 1, \frac{1}{3}\right), \quad H_{1}:\left(1,2,-\frac{1}{2}\right), \quad H_{2}:\left(1,2, \frac{1}{2}\right) .
\end{array}
$$


Then, the superpotential is written as

$$
W=\epsilon_{a b}\left[\left(Y_{E}\right)_{i j} L_{i}^{b} H_{1}^{a} \bar{E}_{j}+\left(Y_{D}\right)_{i j} Q_{i}^{b x} H_{1}^{a} \bar{D}_{j x}+\left(Y_{U}\right)_{i j} Q_{i}^{a x} H_{2}^{b} \bar{U}_{j x}+\mu H_{1}^{b} H_{2}^{a}\right]
$$

Throughout this section, we denote an $S U(3)$ colour index of the fundamental representation by $x, y, z=1,2,3$. The $S U(2)_{L}$ fundamental representation indices are denoted by $a, b, c=1,2$ and the generation indices by $i, j, k=1,2,3 . \epsilon_{a b}=\epsilon^{a b}$ is the totally antisymmetric tensor, with $\epsilon_{12}=1$. Note that the sign of $\mu$ is identical to the one in ISASUGRA [5], but is in the opposite convention to ref. [3]. Presently, real Yukawa couplings only are included. All MSSM running parameters are in the $\overline{D R}$ scheme. The Higgs vacuum expectation values (VEVs) are $\left\langle H_{i}^{0}\right\rangle=v_{i} / \sqrt{2}$ and $\tan \beta=v_{2} / v_{1} \cdot g_{i}$ are the MSSM $\overline{D R}$ gauge couplings and $g_{1}$ is defined in the Grand Unified normalisation $g_{1}=\sqrt{5 / 3} g^{\prime}$, where $g^{\prime}$ is the Standard Model hypercharge gauge coupling. Elements of fermion mass matrices are given by

$$
\left(m_{u}\right)_{i j}=\frac{1}{\sqrt{2}}\left(Y_{U}\right)_{i j} v_{2}, \quad\left(m_{d, e}\right)_{i j}=\frac{1}{\sqrt{2}}\left(Y_{D, E}\right)_{i j} v_{1}
$$

for the up quark, down quark and charged lepton matrices respectively.

\subsection{SUSY Breaking Parameters}

We now tabulate the notation of the soft SUSY breaking parameters. The trilinear scalar interaction potential is

$$
V_{3}=\epsilon_{a b}\left[\tilde{Q}_{i_{L}}^{x a}\left(U_{A}\right)_{i j} \tilde{u}_{j x_{R}} H_{2}^{b}+\tilde{Q}_{i_{L}}^{x b}\left(D_{A}\right)_{i j} \tilde{d}_{j x_{R}} H_{1}^{a}+\tilde{L}_{i_{L}}^{b}\left(E_{A}\right)_{i j} \tilde{e}_{j_{R}} H_{1}^{a}+H . c .\right],
$$

where fields with a tilde are the scalar components of the superfield with the identical capital letter. Also,

$$
\left(A_{U, D, E}\right)_{i j}=\left(U_{A}, D_{A}, E_{A}\right)_{i j} /\left(Y_{U, D, E}\right)_{i j}
$$

(no summation on $i, j$ ) are often referred to in the literature as soft $A$-parameters.

The scalar bilinear SUSY breaking terms are contained in the potential

$$
\begin{aligned}
V_{2}= & m_{H_{1}}^{2} H_{1 a}{ }^{*} H_{1}^{a}+m_{H_{2}}^{2} H_{2 a}{ }^{*} H_{2}^{a}+\tilde{Q}_{i x a}^{*}\left(m_{\tilde{Q}}^{2}\right)_{i j} \tilde{Q}_{j}^{x a}+\tilde{L}_{i a}^{*}\left(m_{\tilde{L}}^{2}\right)_{i j} \tilde{L}_{j}^{a}+ \\
& \tilde{u}_{i}^{x}\left(m_{\tilde{u}}^{2}\right)_{i j} \tilde{u}_{j x}^{*}+\tilde{d}_{i}^{x}\left(m_{\tilde{d}}^{2}\right)_{i j} \tilde{d}_{j x}^{*}+\tilde{e}_{i}\left(m_{\tilde{e}}^{2}\right)_{i j} \tilde{e}_{j}^{*}+\epsilon_{a b}\left(m_{3}^{2} H_{2}^{a} H_{1}^{b}+H . c .\right) .
\end{aligned}
$$

For a comparison of these conventions with other popular ones in the literature, see Table 2.2. In the table, we compare the SOFTSUSY conventions with the SUSY Les Houches Accord [29] and ref. [31] (Martin and Vaughn).

Writing the bino as $\tilde{b}, \tilde{w}^{A=1,2,3}$ as the unbroken-SU(2) $L$ gauginos and $\tilde{g}^{X=1 \ldots 8}$ as the gluinos, the gaugino mass terms are contained in the Lagrangian

$$
\mathcal{L}_{G}=\frac{1}{2}\left(M_{1} \tilde{b} \tilde{b}+M_{2} \tilde{w}^{A} \tilde{w}^{A}+M_{3} \tilde{g}^{X} \tilde{g}^{X}\right)+\text { h.c. }
$$

\subsection{Tree-Level Masses}

Here we suppress any gauge indices and follow the notation of ref. [3] closely. The Lagrangian contains the neutralino mass matrix as $-\frac{1}{2} \tilde{\psi}^{0 T} \mathcal{M}_{\tilde{\psi}^{0}} \tilde{\psi}^{0}+$ h.c., where $\tilde{\psi}^{0}=\left(-i \tilde{b},-i \tilde{w}^{3}, \tilde{h}_{1}, \tilde{h}_{2}\right)^{T}$ and

$$
\mathcal{M}_{\tilde{\psi}^{0}}=\left(\begin{array}{cccc}
M_{1} & 0 & -M_{Z} c_{\beta} s_{W} & M_{Z} s_{\beta} s_{W} \\
0 & M_{2} & M_{Z} c_{\beta} c_{W} & -M_{Z} s_{\beta} c_{W} \\
-M_{Z} c_{\beta} s_{W} & M_{Z} c_{\beta} c_{W} & 0 & -\mu \\
M_{Z} s_{\beta} s_{W} & -M_{Z} s_{\beta} c_{W} & -\mu & 0
\end{array}\right) \text {. }
$$




\begin{tabular}{ccc} 
SOFTSUSY & SLHA & Martin and Vaughn \\
\hline$Y^{U}$ & $Y^{U}$ & $\left(Y^{U}\right)^{T}$ \\
$Y^{D, E}$ & $Y^{D, E}$ & $\left(-Y^{D, E}\right)^{T}$ \\
$U_{A}$ & $T_{U}$ & $h_{U}^{T}$ \\
$D_{A}, E_{A}$ & $T_{D, E}$ & $\left(-h_{D, E}\right)^{T}$ \\
$m_{\tilde{Q}, \tilde{L}}^{2}$ & $m_{\tilde{Q}, \tilde{L}}^{2}$ & $m_{\tilde{Q}, \tilde{L}}^{2}$ \\
$m_{\tilde{u}, \tilde{d}, \tilde{e}}^{2}$ & $m_{\tilde{u}, \tilde{d}, \tilde{e}}^{2}$ & $m_{\tilde{u}, \tilde{d}, \tilde{e}}^{2}$ \\
$\mu$ & $\mu$ & $\mu$ \\
$m_{3}^{2}$ & $B \mu$ & $B$ \\
$M_{i}$ & $M_{i}$ & $M_{i}$ \\
$m_{H_{1,2}}^{2}$ & $m_{H_{1,2}}^{2}$ & $m_{H_{d, u}}^{2}$
\end{tabular}

Table 1. Comparison of conventions between SOFTSUSY and the literature. Note that simple models of SUSY breaking, for example the CMSSM, will have negative gaugino masses $M_{i}$ in the SOFTSUSY conventions.

We use $s$ and $c$ for sine and cosine, so that $s_{\beta} \equiv \sin \beta, c_{\beta} \equiv \cos \beta$ and $s_{W}\left(c_{W}\right)$ is the sine (cosine) of the weak mixing angle. The 4 by 4 neutralino mixing matrix is an orthogonal matrix $O$ with real entries, such that $O^{T} \mathcal{M}_{\tilde{\psi}^{0}} O$ is diagonal. The neutralinos $\chi_{i}^{0}$ are defined such that their absolute masses increase with increasing $i$. Some of their mass values can be negative.

We make the identification $\tilde{w}^{ \pm}=\left(\tilde{w}^{1} \mp i \tilde{w}^{2}\right) / \sqrt{2}$ for the charged winos and $\tilde{h}_{1}^{-}, \tilde{h}_{2}^{+}$for the charged higgsinos. The Lagrangian contains the chargino mass matrix as $-\tilde{\psi}^{-T} \mathcal{M}_{\tilde{\psi}^{+}} \tilde{\psi}^{+}+$h.c., where $\tilde{\psi}^{+}=$ $\left(-i \tilde{w}^{+}, \tilde{h}_{2}^{+}\right)^{T}, \tilde{\psi}^{-}=\left(-i \tilde{w}^{-}, \tilde{h}_{1}^{-}\right)^{T}$ and

$$
\mathcal{M}_{\tilde{\psi}^{+}}=\left(\begin{array}{cc}
M_{2} & \sqrt{2} M_{W} s_{\beta} \\
\sqrt{2} M_{W} c_{\beta} & \mu
\end{array}\right) .
$$

This matrix is then diagonalised by 2 dimensional rotations through angles $\theta_{L}, \theta_{R}$ in the following manner:

$$
\left(\begin{array}{cc}
c_{\theta_{L}} & s_{\theta_{L}} \\
-s_{\theta_{L}} & c_{\theta_{L}}
\end{array}\right) \mathcal{M}_{\tilde{\psi}^{+}}\left(\begin{array}{cc}
c_{\theta_{R}} & -s_{\theta_{R}} \\
s_{\theta_{R}} & c_{\theta_{R}}
\end{array}\right)=\left(\begin{array}{cc}
m_{\chi_{1}}^{+} & 0 \\
0 & m_{\chi_{2}}^{+}
\end{array}\right)
$$

where $m_{\chi_{i}}^{+}$could be negative, with the mass parameter of the lightest chargino being in the top left hand corner.

At tree level the gluino mass, $m_{\tilde{g}}$, is given by $M_{3}$.

Strong upper bounds upon the inter-generational scalar mixing exist [10] and in the following we assume that such mixings are negligible. The tree-level squark and slepton mass squared values for the family $i$ are found by diagonalising the following mass matrices $\mathcal{M}_{\tilde{f}}^{2}$ defined in the $\left(\tilde{f}_{i L}, \tilde{f}_{i R}\right)^{T}$ basis:

$$
\begin{gathered}
\left(\begin{array}{cc}
\left(m_{\tilde{Q}}^{2}\right)_{i i}+m_{u i}^{2}+\left(\frac{1}{2}-\frac{2}{3} s_{W}^{2}\right) M_{Z}^{2} c_{2 \beta} & m_{u_{i}}\left(\left(A_{U}\right)_{i i}-\mu \cot \beta\right) \\
m_{u_{i}}\left(\left(A_{U}\right)_{i i}-\mu \cot \beta\right) & \left(m_{\tilde{u}}^{2}\right)_{i i}+m_{u_{i}}^{2}+\frac{2}{3} s_{W}^{2} M_{Z}^{2} c_{2 \beta}
\end{array}\right), \\
\left(\begin{array}{cc}
\left(m_{\tilde{Q}}^{2}\right)_{i i}+m_{d_{i}}^{2}-\left(\frac{1}{2}-\frac{1}{3} s_{W}^{2}\right) M_{Z}^{2} c_{2 \beta} & m_{d_{i}}\left(\left(A_{D}\right)_{i i}-\mu \tan \beta\right) \\
m_{d_{i}}\left(\left(A_{D}\right)_{i i}-\mu \tan \beta\right) & \left(m_{\tilde{d}}^{2}\right)_{i i}+m_{d_{i}}^{2}-\frac{1}{3} s_{W}^{2} M_{Z}^{2} c_{2 \beta}
\end{array}\right), \\
\left(\begin{array}{cc}
\left(m_{\tilde{L}}^{2}\right)_{i i}+m_{e_{i}}^{2}-\left(\frac{1}{2}-s_{W}^{2}\right) M_{Z}^{2} c_{2 \beta} & m_{e_{i}}\left(\left(A_{E}\right)_{i i}-\mu \tan \beta\right) \\
m_{e_{i}}\left(\left(A_{E}\right)_{i i}-\mu \tan \beta\right) & \left(m_{\tilde{e}}^{2}\right)_{i i}+m_{e_{i}}^{2}-s_{W}^{2} M_{Z}^{2} c_{2 \beta}
\end{array}\right),
\end{gathered}
$$


$m_{f}, e_{f}$ are the mass and electric charge of fermion $f$ respectively. The mixing of the first two families is suppressed by a small fermion mass, which we approximate to zero. The sfermion mass eigenstates are given by

$$
\left(\begin{array}{cc}
m_{\tilde{f}_{1}} & 0 \\
0 & m_{\tilde{f}_{2}}
\end{array}\right)=\left(\begin{array}{cc}
c_{f} & s_{f} \\
-s_{f} & c_{f}
\end{array}\right) \mathcal{M}_{\tilde{f}}^{2}\left(\begin{array}{cc}
c_{f} & -s_{f} \\
s_{f} & c_{f}
\end{array}\right)
$$

where $c_{f}$ is the cosine of the sfermion mixing angle, $\cos \theta_{f}$, and $s_{f}$ the sine. $\theta_{f}$ are set in the convention that the two mass eigenstates are in no particular order and $\theta_{f} \in[-\pi / 4, \pi / 4]$. The sneutrinos of one family are not mixed and their masses are given by

$$
m_{\tilde{\nu}_{i}}^{2}=\left(m_{\tilde{L}}^{2}\right)_{i i}+\frac{1}{2} M_{Z}^{2} c_{2 \beta} .
$$

The CP-even gauge eigenstates $\left(H_{1}^{0}, H_{2}^{0}\right)$ are rotated by the angle $\alpha$ into the mass eigenstates $\left(H^{0} h^{0}\right)$ as follows,

$$
\left(\begin{array}{c}
H^{0} \\
h^{0}
\end{array}\right)=\left(\begin{array}{cc}
c_{\alpha} & s_{\alpha} \\
-s_{\alpha} & c_{\alpha}
\end{array}\right)\left(\begin{array}{c}
\frac{1}{\sqrt{2}} \Re H_{1}^{0} \\
\frac{1}{\sqrt{2}} \Re H_{2}^{0}
\end{array}\right) .
$$

$m_{h^{0}}<m_{H^{0}}$ by definition, and $\alpha \in[-\pi / 4,3 \pi / 4]$. The CP-odd and charged Higgs masses are

$$
m_{A^{0}}^{2}=m_{3}^{2}(\tan \beta+\cot \beta), \quad m_{H^{ \pm}}^{2}=m_{A^{0}}^{2}+M_{W}^{2}
$$

at tree level.

\section{Calculation}

We now show the algorithm used to perform the calculation. Standard Model parameters (fermion and gauge boson masses, the fine structure constant $\alpha\left(M_{Z}\right)$, the Fermi constant from muon decay $G_{F}^{\mu}$ and $\left.\alpha_{3}\left(M_{Z}\right)\right)$ are used as constraints. The soft SUSY breaking parameters and the superpotential parameter $\mu$ are then the free parameters. However, in what follows, $|\mu|$ is constrained by $M_{Z}$ and tan $\beta$ is traded for $m_{3}$ as an input parameter. Therefore, the total list of unconstrained input parameters is: any fundamental soft SUSY breaking parameters (except $m_{3}^{2}$ ), $\tan \beta$ and the $\operatorname{sign}$ of $\mu$. First we describe the evolution of the low-energy Standard Model input parameters below $M_{Z}$, then detail the rest of the algorithm.

\subsection{Below $M_{Z}$}

$\alpha\left(M_{Z}\right), \alpha_{s}\left(M_{Z}\right)$ are first evolved to $1 \mathrm{GeV}$ using 3 loop QCD and 1 loop QED [11-13] with stepfunction decoupling of fermions at their running masses. We have checked that the contribution from 2-loop matching [14] is negligible; the effect of 3-loop terms in the renormalisation group equations is an order of magnitude larger. Then, the two gauge couplings and all Standard Model fermion masses except the top mass are run to $M_{Z}$. The $\beta$ functions of fermion masses are taken to be zero at renormalisation scales below their running masses. The parameters at $M_{Z}$ are used as the low energy boundary condition in the rest of the evolution.

\subsection{Initial Estimate}

The algorithm proceeds via the iterative method, and therefore an approximate initial guess of MSSM parameters is required. For this, the third family $\overline{D R}$ Yukawa couplings are approximated by

$$
h_{t}(Q)=\frac{m_{t}(Q) \sqrt{2}}{v \sin \beta}, \quad h_{b, \tau}(Q)=\frac{m_{b, \tau}(Q) \sqrt{2}}{v \cos \beta}
$$




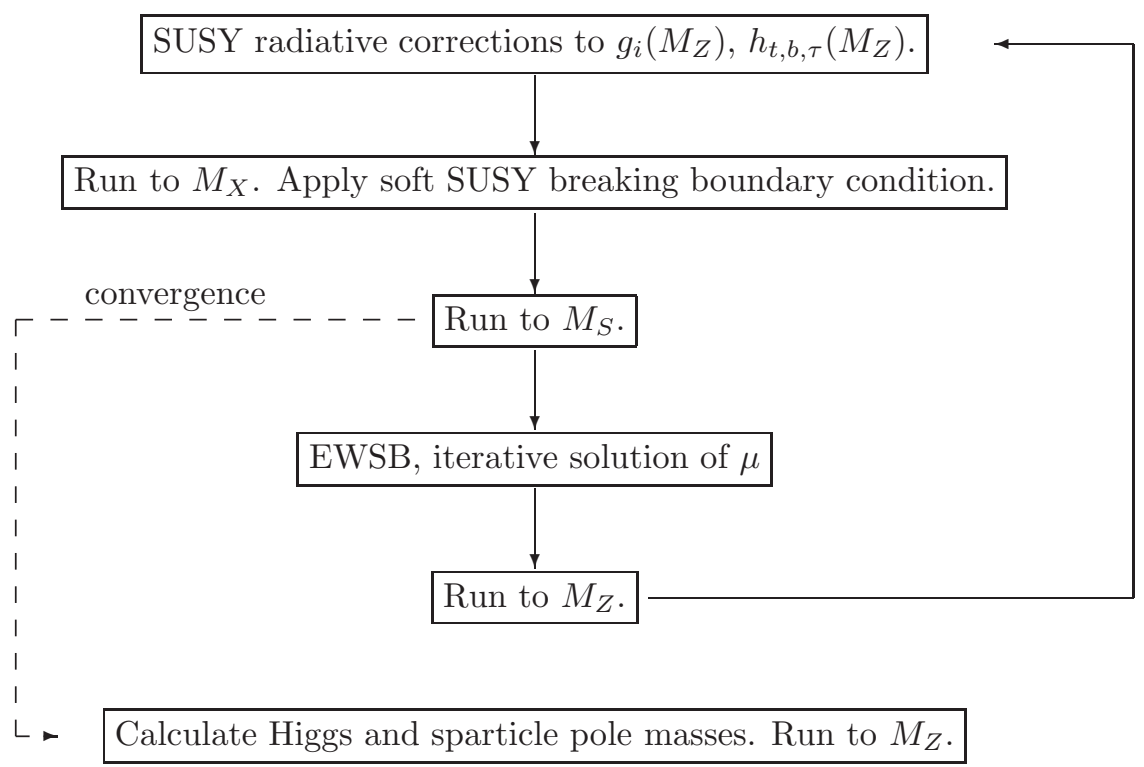

Figure 1. Iterative algorithm used to calculate the SUSY spectrum. Each step (represented by a box) is detailed in the text. The initial step is the uppermost one. $M_{S}$ is the scale at which the EWSB conditions are imposed, as discussed in the text. $M_{X}$ is the scale at which the high energy SUSY breaking boundary conditions are imposed.

where $v=246.22 \mathrm{GeV}$ is the Standard Model Higgs VEV and $Q=m_{t}\left(m_{t}\right)$ is the renormalisation scale. The $\overline{M S}$ values of fermion masses are used for this initial estimate. The fermion masses and $\alpha_{s}$ at the top mass are obtained by evolving the previously obtained fermion masses and gauge couplings from $M_{Z}$ to $m_{t}$ (with the same accuracy). The electroweak gauge couplings are estimated by $\alpha_{1}\left(M_{Z}\right)=5 \alpha\left(M_{Z}\right) /\left(3 c_{W}^{2}\right), \alpha_{2}\left(M_{Z}\right)=\alpha\left(M_{Z}\right) / s_{W}^{2}$. Here, $s_{W}$ is taken to be the on-shell value. These two gauge couplings are then evolved to $m_{t}$ with 1-loop Standard Model $\beta$ functions, including the effect of a light higgs (without decoupling it). In this initial guess, no SUSY threshold effects are calculated. The gauge and Yukawa couplings are then evolved to the unification scale $M_{X}$ with the one-loop MSSM $\beta$ functions, where the user-supplied boundary condition on the soft terms is applied. Also, $\mu\left(M_{X}\right)=\operatorname{sgn}(\mu) \times 1 \mathrm{GeV}$ and $m_{3}\left(M_{X}\right)=0$ are imposed. These initial values are irrelevant; they are overwritten on the next iteration by more realistic boundary conditions. $\mu\left(M_{X}\right)$ is set to be of the correct sign because its sign does not change through renormalisation.

The whole system of MSSM soft parameters and SUSY couplings is then evolved to 1-loop order to $M_{Z}$. At $M_{Z}$, the tree-level electroweak symmetry breaking (EWSB) conditions are applied [6] to predict $\mu$ and $m_{3}$. The masses and mixings of MSSM super particles are then calculated at treelevel order by using the SUSY parameters (and $m_{3}$ ) calculated at $M_{Z}$. The resulting set of MSSM parameters is then used as the initial guess for the iterative procedure described below.

\subsection{Gauge and Yukawa Couplings}

Figure 3.2 shows the iterative procedure, starting from the the top. The whole calculation is currently performed in the real full three family approximation, i.e. all Yukawa couplings are set to be real, but quark mixing is incorporated. First of all, the one-loop radiative corrections are applied to the gauge and third-family Yukawa couplings. For these, we rely heavily on ref. [3] by Bagger, Matchev, Pierce 
and Zhang (BMPZ) $)^{2}$. In the threshold corrections, we use running $\overline{D R}$ masses and parameters at the relevant scale, unless denoted otherwise in the text. $m_{t}\left(M_{Z}\right)$ is calculated with 2-loop QCD [15] and the full one-loop supersymmetric contributions to $m_{t}\left(M_{Z}\right)$, including logarithmic and finite contributions (eqs. (D.16)-(D.18) of BMPZ):

$$
\begin{aligned}
m_{t}\left(M_{Z}\right)_{M S S M}^{\overline{D R}}= & \Sigma_{t}^{B M P Z}+m_{t}^{\text {pole }}\left(1-\frac{\alpha_{s}\left(M_{Z}\right)}{3 \pi}(5-3 L)+\right. \\
& \left.\alpha_{s}^{2}\left(M_{Z}\right)\left(-0.538+\frac{43}{24 \pi^{2}} L-\frac{3}{8 \pi^{2}} L^{2}\right)\right),
\end{aligned}
$$

where $L \equiv \ln \left(m_{t}^{2}\left(M_{Z}\right)_{M S S M}^{\overline{D R}} / M_{Z}^{2}\right)$. We denote the BPMZ corrections without the one-loop QCD part as $\Sigma_{t}^{B M P Z}$. These corrections are necessary because the region of valid EWSB is very sensitive to $m_{t}[6]$. To calculate $m_{b}\left(M_{Z}\right)^{\overline{D R}}$, we first calculate the Standard Model $\overline{D R}$ value from the $\overline{M S}$ one $[15,32]$

$$
m_{b}\left(M_{Z}\right)_{S M}^{\overline{D R}}=m_{b}\left(M_{Z}\right)_{S M}^{\overline{M S}}\left(1-\frac{\alpha_{s}^{\overline{D R}}}{3 \pi}-\frac{23 \alpha_{s}^{\overline{D R}^{2}}}{72 \pi^{2}}+\frac{3 g^{2}}{128 \pi^{2}}+\frac{13 g_{1}^{2}}{1152 \pi^{2}}\right) .
$$

We then add the leading one-loop supersymmetric corrections

$$
m_{b}\left(M_{Z}\right)_{M S S M}^{\overline{D R}}=m_{b}\left(M_{Z}\right)_{S M}^{\overline{D R}} /\left(1+\Delta_{S U S Y}^{b}\right) .
$$

The contributions to $\Delta_{S U S Y}^{b}$ are included in full from eq. D.18 of BMPZ (neglecting the term proportional to $e$, since that is already included in the QED calculation of the SM $m_{b}\left(M_{Z}\right)$ ). Both finite and leading logarithmic corrections are included. After the Standard Model $\overline{M S}$ bar of $m_{\tau}$ is converted to the $\overline{D R}$ value via

$$
m_{\tau}\left(M_{Z}\right)_{S M}^{\overline{D R}}=m_{\tau}\left(M_{Z}\right)_{S M}^{\overline{M S}}\left(1-\frac{3}{128 \pi^{2}}\left(g_{1}^{2}-g_{2}^{2}\right)\right) .
$$

The full one-loop MSSM corrections from the appendix of BPMZ (aside from the photon contribution, since that has already been included in $\left.m_{\tau}\left(M_{Z}\right)_{S M}^{\overline{M S}}\right)$ are then used to correct $m_{\tau}\left(M_{Z}\right)_{S M}^{\overline{D R}}$ :

$$
m_{\tau}\left(M_{Z}\right)_{M S S M}^{\overline{D R}}=m_{\tau}\left(M_{Z}\right)_{S M}^{\overline{D R}}\left(1+\Sigma_{\tau}\right) .
$$

The one-loop $\overline{D R}$ values for $m_{t}\left(M_{Z}\right), m_{b}\left(M_{Z}\right), m_{\tau}\left(M_{Z}\right)$ are then substituted with the $\overline{D R}$ value of $v(Q)$ into eq. (2.3) to calculate the third family $\overline{D R}$ Yukawa couplings at $M_{Z} \cdot v(Q)$ is run to two-loops. The other diagonal elements of the Yukawa matrices are set by eq. (2.3) but with fermion masses replaced by the $\overline{M S}$ values.

The default option is to perform the calculation in the dominant third-family approximation, where all elements of Yukawa matrices expect for the $(3,3)$ elements are set to zero. There are also options described in appendix D for performing the calculation in the unmixed 3-family approximation or the fully-mixed 3-family case. If a flavour-mixing option is chosen (see section E.9), the Yukawa couplings are then mixed using the "standard parameterisation" of the CKM matrix [17] with CPviolating phase set either to zero or $\pi$, whichever results in a positive entry for $\left(V_{C K M}\right)_{13}$ (also known as $\left.V_{u b}\right)$ :

$$
V_{C K M}=\left(\begin{array}{ccc}
c_{12} c_{13} & s_{12} c_{13} & p s_{13} \\
-s_{12} c_{23}-p c_{12} s_{23} s_{13} & c_{12} c_{23}-p s_{12} s_{23} s_{13} & s_{23} c_{13} \\
s_{12} s_{23}-p c_{12} c_{23} s_{13} & -c_{12} s_{23}-p s_{12} c_{23} s_{13} & c_{23} c_{13}
\end{array}\right),
$$

\footnotetext{
${ }^{2}$ Whenever a reference to an equation in BMPZ is made, it is understood that the sign of $\mu$ must be reversed.
} 
where $s_{i j} \equiv \sin \theta_{i j}, c_{i j} \equiv \cos \theta_{i j}$ and $p= \pm 1$. Sign conventions are automatically chosen such that diagonal entries and entries above the diagonal in $V_{C K M}$ are positive. Note that $V_{C K M}$ is a member of $O(3)$, i.e. $V_{C K M}^{-1}=V_{C K M}^{T}$. While complex phase effects are obviously not taken into account in eq. 3.7, it is hoped that the magnitudes of the main quark mixing effects will be. In fact, using the central values for $\left|\left(V_{C K M}\right)_{i j}\right|$ given by the particle data group [17], the magnitudes of all elements in $V_{C K M}$ in the first row and last column may be exactly reproduced. Of the other entries, $\left|V_{c s}\right|$ is accurate at the $2 \times 10^{-5}$ level and $\left|V_{c d}\right|$ at the $4 \times 10^{-4}$ level: surely negligible for most practical purposes. $\left|V_{t s}\right|$ is also quite accurate (to $1.2 \%$ ), but it should be noted that $\left|V_{t d}\right|$ is wrong by around $50 \%$. Any flavour physics effects sensitive to $\left|V_{t d}\right|$ is therefore subject to this large uncertainty on its value issuing from SOFTSUSY ${ }^{3}$. The up (by default), or down Yukawa couplings at $M_{Z}$ are mixed in the weak eigenbasis via

$$
\left(Y_{U}\right)^{\prime}=V_{C K M}^{T}\left(Y^{U}\right) V_{C K M}, \quad\left(Y_{D}\right)^{\prime}=V_{C K M}\left(Y^{D}\right) V_{C K M}^{T}
$$

where the primed Yukawa matrix is in the weak eigenbasis and the unprimed is in the mass eigenbasis.

Full one-loop corrections to $g_{i}\left(M_{Z}\right)$ are included. The treatment of electroweak gauge couplings follows from appendix $\mathrm{C}$ of BMPZ, and includes: two-loop corrections from the top, electroweak boson and the lightest CP-even Higgs. The pole value of $m_{t}$ is used in the calculation of the $W$ and $Z$ self energy in order to calculate $\sin \theta_{w}$, since this is what is assumed in the two-loop corrections. We use the fine structure constant $\alpha\left(M_{Z}\right)^{\overline{M S}}$, the Z-boson mass $M_{Z}$ and the Fermi decay constant $G_{\mu}$ as inputs. $M_{W}$ is predicted from these inputs. Because the EWSB constraints tend to depend sensitively upon $g_{1,2}\left(M_{Z}\right)$, accurate values for them are determined iteratively. An estimate of the $\overline{D R}$ value of $s_{W}^{2}$ is used to yield a better estimate until the required accuracy is reached (usually within 3 or 4 iterations). The QCD coupling is input as $\alpha_{s}\left(M_{Z}\right)^{\overline{M S}}$ and is modified by gluino, squark and top loops as in eqs. (2),(3) of BMPZ in order to obtain the MSSM $\overline{D R}$ value.

\subsection{MSSM Renormalisation}

All soft breaking and SUSY parameters are then evolved to the scale

$$
M_{S} \equiv \sqrt{m_{\tilde{t}_{1}}\left(M_{S}\right) m_{\tilde{t}_{2}}\left(M_{S}\right)}
$$

where [18] the scale dependence of the electroweak breaking conditions is smallest. Throughout the iteration described here, the renormalisation group evolution (RGE) employs three family, 2-loop MSSM $\beta$ functions for the supersymmetric parameters [2]. $\tan \beta$ and the Higgs VEV parameter $v$ are also run to two-loop order in the Feynman gauge, although the Higgs VEV RGE is missing terms $\mathcal{O}\left(g_{2}^{4}, g_{2}^{2} g_{1}^{2}, g_{1}^{4}\right) /\left(16 \pi^{2}\right)^{2}[19,20]$. There is no step-function decoupling of sparticles: this is taken into account at leading logarithmic order in the radiative corrections previously calculated at $M_{Z}$ and in the calculation of the physical sparticle spectrum at $M_{S}$, described below. All $\beta$ functions are real and include 3 family (and mixing) contributions. If no flavour mixing is present in the model specified by the user, the 2-loop parts of the RGEs switch to the dominant 3rd-family version, where the lighter two families' Yukawa couplings are neglected.

\subsection{Electroweak Symmetry Breaking}

The Higgs VEV parameter $v\left(M_{S}\right)$ is set by:

$$
v^{2}\left(M_{S}\right)=4 \frac{M_{Z}^{2}+\Re \Pi_{Z Z}^{T}\left(M_{S}\right)}{g_{2}^{2}\left(M_{S}\right)+3 g_{1}^{2}\left(M_{S}\right) / 5},
$$

\footnotetext{
${ }^{3}$ It is hoped in the future to include complex phases in Yukawa matrices, sfermion soft mass squared terms and trilinear scalar couplings.
} 
where $M_{Z}$ is the pole $Z$ mass and $\Pi_{Z Z}^{T}$ is the transverse $Z$ self-energy. The full one-loop EWSB conditions at this scale are then employed to ${ }^{4}$ calculate $m_{3}\left(M_{S}\right)$ and $\mu\left(M_{S}\right) . \mu\left(M_{S}\right)$ requires an iterative solution because the tadpoles depend upon the value of $\mu$ assumed. The symmetry breaking condition for $\mu$ can be phrased as [3]

$$
\mu^{2}=\frac{1}{2}\left(\tan 2 \beta\left[m_{\bar{H}_{2}}^{2} \tan \beta-m_{\bar{H}_{1}}^{2} \cot \beta\right]-M_{\bar{Z}}^{2}\right),
$$

where $m_{\bar{H}_{i}}^{2}=m_{H_{i}}^{2}-t_{i} / v_{i}, M_{\bar{Z}}^{2}=M_{Z}^{2}+\Re \Pi_{Z Z}^{T}\left(M_{Z}^{2}\right)$ is the running $Z$ mass and $t_{i}$ are the tadpole contributions. The value of $\mu$ coming from the tree-level EWSB condition (eq. 3.11, with $\Re \Pi_{Z Z}^{T}=$ $\left.t_{i}=0\right)$ is utilised as an initial guess, then the one-loop contributions in the tadpoles and self-energy terms are added to provide a new value of $\mu\left(M_{S}\right)$. Two-loop terms $\mathcal{O}\left(\alpha_{t}^{2}\right), \mathcal{O}\left(\alpha_{b} \alpha_{\tau}\right), \mathcal{O}\left(\alpha_{b}^{2}\right), \mathcal{O}\left(\alpha_{b} \alpha_{s}\right)$, $\mathcal{O}\left(\alpha_{t} \alpha_{s}\right), \mathcal{O}\left(\alpha_{\tau}^{2}\right)$ and $\mathcal{O}\left(\alpha_{t} \alpha_{b}\right)$ are also included in the tadpoles [21, 26]. The tadpole corrections are then calculated using the new value of $\mu\left(M_{S}\right)$ and the procedure is repeated until it converges to a given accuracy. $m_{3}\left(M_{S}\right)$ is then determined by input the value of $\mu\left(M_{S}\right)$ into the EWSB condition

$$
m_{3}^{2}=\frac{s_{2 \beta}}{2}\left(m_{\bar{H}_{1}}^{2}+m_{\bar{H}_{2}}^{2}+2 \mu^{2}\right) .
$$

The ensemble of MSSM parameters are then evolved using the $\beta$ functions described above to the user supplied scale $M_{X}$. If gauge-unification has been specified as a boundary condition, the current estimate of $M_{X}$ is revised to leading log order to provide a more accurate value upon the next iteration:

$$
M_{X}^{\text {new }}=M_{X} \exp \left(\frac{g_{2}\left(M_{X}\right)-g_{1}\left(M_{X}\right)}{g_{1}^{\prime}\left(M_{X}\right)-g_{2}^{\prime}\left(M_{X}\right)}\right)
$$

where primes denote derivatives calculated to 2-loop order. The user-supplied boundary conditions are then imposed upon the soft terms before the model is evolved back down to $M_{S}$. The super particle mass spectrum is determined at this scale. Because $\mu$ and $m_{3}$ are more scale independent at $M_{S}$ as opposed to some other scale, the Higgs, neutralino and chargino masses also ought to be more scale independent by determining them at this scale.

\subsection{MSSM Spectrum}

In the following description of the approximations involved in the calculation of the super particle spectrum, it is implicit that where masses appear, their $\overline{D R}$ values are employed. The running value of $s_{W}(\mu)=e(\mu) / g_{2}(\mu)$ is also employed. In loop corrections to sparticle masses, the Yukawa couplings of the first two families are set to zero, being highly suppressed compared to those of the third family. All sparticle masses are calculated with the full SUSY one-loop BPMZ corrections at the scale $M_{S U S Y}$. Most sparticle masses are calculated at external momenta equal to their $\overline{D R}$ mass $m\left(M_{S U S Y}\right)$.

The physical gluino mass is calculated to full one-loop order as follows. The running parameters are evaluated at renormalisation scale $\mu=M_{S U S Y}$ and external momentum $p=M_{3}(\mu)$ in the following corrections:

$$
\begin{aligned}
\Delta_{\tilde{g}}(\mu)=\frac{g_{3}(\mu)^{2}}{16 \pi^{2}} & \left(15+9 \ln \left(\frac{\mu^{2}}{p^{2}}\right)-\sum_{q} \sum_{i=1}^{2} B_{1}\left(p, m_{q}, m_{\tilde{q}_{i}}, \mu\right)+\right. \\
& \left.\sum_{q=t, b} \frac{m_{q}}{M_{3}(\mu)} s_{2 \theta_{q}}\left[B_{0}\left(p, m_{q}, m_{\tilde{q}_{1}}, \mu\right)-B_{0}\left(p, m_{q}, m_{\tilde{q}_{2}}, \mu\right)\right]\right) .
\end{aligned}
$$

\footnotetext{
${ }^{4}$ Note that there is also an option to extract $m_{H_{1}}\left(M_{S U S Y}\right)$ and $m_{H_{2}}\left(M_{S U S Y}\right)$ from input $\mu\left(M_{S U S Y}\right)$ and $m_{A}$ (pole) values, see section E.8.
} 
The Passarino-Veltman functions $B_{0,1}$ are given in appendix B of BMPZ. The physical gluino mass is then given by

$$
m_{\tilde{g}}=M_{3}\left(M_{S U S Y}\right)\left(1+\Delta_{\tilde{g}}\left(M_{S U S Y}\right)\right)
$$

The gluino mass is allowed to be negative, as is the case in mAMSB, for example. Of course the kinematic mass is just the absolute value, and the phase may be rotated away, altering the phases of some of the Feynman rules. Negative masses for neutralinos can also be rotated away in this way.

For the mixed neutralinos and charginos, the external momentum is equal to the $\overline{D R}$ value of the diagonalised mass. All mixing angles and matrices are defined such that they diagonalise the one-loop corrected mass matrix evaluated at the minimum $\overline{D R}$ mass eigenvalue. In the case where inter-family flavour mixing is not used in the sfermion sector, the above is also how the sfermion masses and third family mixing is calculated. When flavour mixing between families is used, at tree-level, the full flavour structure is present in sfermion masses. Loop corrections are added only to the same family entries of the mass squared matrix, not to intra-family mixing entries. For the first two families, the external momentum is set equal to the $\overline{D R}$ sfermion mass itself. For the third family, the external momentum is set equal to the the mass of the lightest $\overline{D R}$ mass eigenvalue of the third family sfermion of the particular flavour in question. All of the SOFTSUSY loop corrections also neglect intra-family mixing themselves.

The pseudo-scalar Higgs mass $m_{A^{0}}$ and CP-even Higgs masses $m_{h^{0}}, m_{H^{0}}$ are determined to full one-loop order as in eq. (E.6) of BMPZ in order to reduce their scale dependence, which can be large [22]. The zero-momentum $\mathcal{O}\left(\alpha_{t}^{2}\right), \mathcal{O}\left(\alpha_{b} \alpha_{\tau}\right), \mathcal{O}\left(\alpha_{b}^{2}\right), \mathcal{O}\left(\alpha_{b} \alpha_{s}\right), \mathcal{O}\left(\alpha_{t} \alpha_{s}\right), \mathcal{O}\left(\alpha_{\tau}^{2}\right), \mathcal{O}\left(\alpha_{t} \alpha_{b}\right) 2-$ loop corrections are also included in $m_{A^{0}}, m_{h^{0}}, m_{H^{0}}$ [23-26]. All one-loop corrections are included in the determination of the charged Higgs pole mass. Every Higgs mass is determined at an external momentum scale equal to its $\overline{D R}$ mass.

Finally, the running MSSM parameters are evolved back down to $M_{Z}$. The whole process is iterated as shown in figure 3.2, until the $\overline{D R}$ sparticle masses evaluated at $M_{S}$ all converge to better than the desired fractional accuracy (TOLERANCE), which may be set by the user in the main program or input file.

\subsection{Fine Tuning}

We now detail the fine-tuning calculation. As lower bounds on super partner masses are pushed up by colliders, $m_{H_{1}}$ and $m_{H_{2}}$ may be forced to be much larger than $M_{Z}$ if they are related to the other super particle masses, as is the case for example in the case of minimal super gravity. If we re-phrase eq. (3.11) as

$$
M_{\bar{Z}}^{2}=-2 \mu^{2}+\tan 2 \beta\left[m_{\bar{H}_{2}}^{2} \tan \beta-m_{\bar{H}_{1}}^{2} \cot \beta\right],
$$

we see that the terms on the right-hand side must have some degree of cancellation in order to reproduce the observed value of $M_{Z}$. But $\mu$ has a different origin to the SUSY breaking parameters and the balancing appears unnatural. Various measures have been proposed in order to quantify the apparent cancellation, for example ref.s $[27,28]$. The definition of naturalness $c_{a}$ of a 'fundamental' parameter $a$ employed here is [28]

$$
c_{a} \equiv\left|\frac{\partial \ln M_{Z}^{2}}{\partial \ln a}\right| .
$$

From a choice of a set of fundamental parameters defined at the scale $M_{X}:\left\{a_{i}\right\}$, the fine-tuning of a particular model is defined to be $c=\max \left(c_{a}\right) .\left\{a_{i}\right\}$ are any parameters in the user supplied boundary condition on the soft supersymmetry breaking parameters augmented by $h_{t}\left(M_{X}\right), \mu\left(M_{X}\right)$ 
and $m_{3}\left(M_{X}\right)$. The derivatives in eq. (3.17) are calculated by numerically finding the derivative of $M_{Z}^{\text {pole }}=\hat{M}_{Z}+\Re \Pi_{Z Z}^{T}\left(M_{Z}^{2}\right)$ in eq. (3.11). The input parameters are changed slightly (one by one), then the MSSM parameter ensemble is run from $M_{X}$ to $M_{S}$ where the sparticle mass spectrum is determined along with the corresponding $\overline{M S}$ Higgs VEV parameter $v^{2} \equiv v_{1}^{2}+v_{2}^{2}$. First of all, $\tan \beta\left(M_{S}\right)$ is determined by inverting eq. (3.12) and the resulting value is utilised in a version of eq. (3.11) inverted to give $M_{Z}^{\text {pole }}$ in terms of the other parameters. The resulting value of $M_{Z}^{\text {pole }}$ is the prediction for the new changed input parameters, and its derivative is determined by examining its behaviour as the initial changes in input parameters tend to zero.

\section{A Running SOFTSUSY}

A main program which produces an executable called softpoint.x, is included in the SOFTSUSY distribution. For the calculation of the spectrum of single points in parameter space, we recommend the SLHA [29] input/output option. The user must modify a file (e.g. lesHouchesInput, as provided in the standard distribution) that specifies the input parameters. The user may then run the code with

./softpoint.x leshouches < lesHouchesInput

In this case, the output will also be in SLHA format. Such output can be used for input into other programs which subscribe to the accord, such as SDECAY [35], PYTHIA [36] (for simulating sparticle production and decays at colliders) or micrOMEGAs [37] (for calculating the relic density of neutralinos, $b \rightarrow s \gamma$ and $\mu \rightarrow e \gamma$ ), for example. For further details on the necessary format of the input file, see ref. [29] and appendix A.1.

If the user desires to quickly run a single parameter point in AMSB, mSUGRA or GMSB parameter space, but does not wish to use the SLHA, the following options are available:

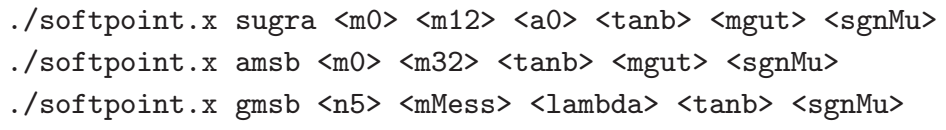

Bracketed entries should be replaced by the desired numerical values, (in $\mathrm{GeV}$ if they are dimensionful). The program will provide output from one point in CMSSM, mAMSB or GMSB. If <mgut> is specified as unified on input, SOFTSUSY will determine mgut to be the scale that $g_{1}\left(M_{G U T}\right)=g_{2}\left(M_{G U T}\right)$, usually of order $10^{16} \mathrm{GeV}$. If <mgut> is set to be msusy, the SUSY breaking parameters will be set at $M_{S U S Y}$.

For users that are not familiar with $\mathrm{C}++^{+}$, we note that the executable interface allows the calculation at just one parameter point in SUSY breaking space. If scans are required, the user can either call SOFTSUSY from a shell script or use a system call from a main $\mathrm{C}$ program to the executable. Alternatively, a main program showing an example of a scan is provided. $\mathrm{C}^{++}$beginners should note in the following that "method" means function, that objects contain a list of data structures and functions and that for a user to access (change or reference) the data encoded in an object, one of its functions should be called. Such functions are given in tables of the following appendices.

\section{A.1 Input file}

If, as recommended, the SLHA option is used for input, the user may add a SOFTSUSY-specific block to the input file in the following format, with bracketed entries replaced by double precision values:

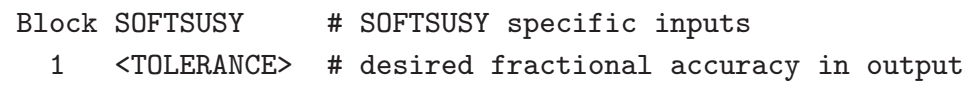




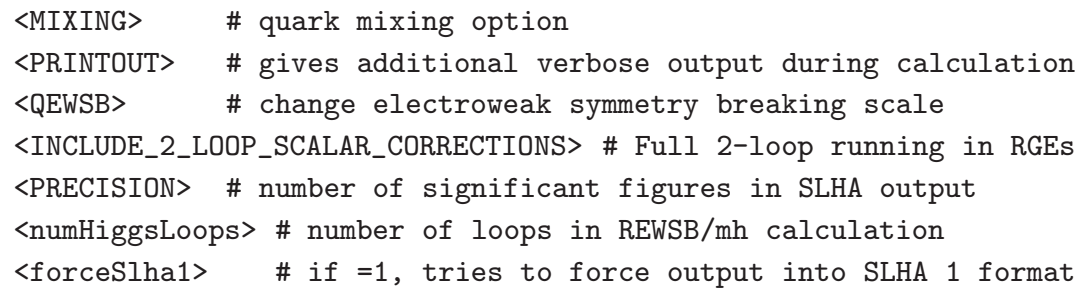

The fractional numerical precision on masses and couplings output by SOFTSUSY is better than TOLERANCE, which sets the accuracy of the whole calculation. The iteration of each physical SUSY particle mass is required to converge to a fractional accuracy smaller than TOLERANCE. Sub-iterations are required to converge to a better accuracy than $10^{-2} \times$ TOLERANCE for $s_{W}$ and $10^{-4} \times$ TOLERANCE for $\mu$. The accuracy of the Runge-Kutta RGE changes from iteration to iteration but is proportional to the value of TOLERANCE. Values between $10^{-2}$ and $10^{-6}$ are common, lower values mean that SOFTSUSY takes significantly longer to perform the calculation.

The next parameter MIXING determines what $M_{Z}$ boundary condition will be used for the quark Yukawa matrix parameters. MIXING $=0.0$ sets the quark mixings to zero but includes the first two family's diagonal terms. MIXING $=1.0,2.0$ sets all the mixing at $M_{Z}$ to be in the up-quark or downquark sector respectively, as in eq. (3.8).

Setting PRINTOUT to a non-zero value gives additional information on each successive iteration. If PRINTOUT $>0$, a warning flag is produced when the overall iteration finishes. The predicted values of $M_{Z}^{\text {pole }}$ and $\tan \beta\left(M_{S}\right)$ after iteration convergence are also output ${ }^{5}$. The level of convergence, $\mu\left(M_{S}\right), m_{3}^{2}\left(M_{S}\right)$ and $M_{Z}$ are output with each iteration, as well as a flag if the object becomes nonperturbative. PRINTOUT $>1$ produces output on the fine-tuning calculation. The predicted values of $M_{Z}^{\text {pole }}$ and $\tan \beta\left(M_{S}\right)$ are output with each variation in the initial inputs. A warning flag is produced when a negative-mass squared scalar is present. PRINTOUT $>2$ prints output on the sub-iterations that determine $\mu\left(M_{S}\right)$ and $s_{W}\left(M_{S}\right)$, and flags the nature of any tachyons encountered. Values PRINTOUT $>0$ are only required if additional diagnostics are required for debugging purposes.

QEWSB may be used to multiplicatively change the scale $M_{S}$ at which the Higgs potential is minimised and sparticle masses calculated, or it may alternatively be used as a fixed scale. This can be useful if one wants to examine the scale dependence of the results [30]. Setting QEWSB $=x<M_{Z} / 1$ $\mathrm{GeV}$, sets $M_{S}=x \sqrt{m_{\tilde{t}_{1}}\left(M_{S}\right) m_{\tilde{t}_{2}}\left(M_{S}\right)}$. Values from 0.5 to 2 are common. If $M_{S}<M_{Z}$ results from the above expression, $M_{S}=M_{Z}$ is used. If on the other hand a fixed scale is desired from $M_{S}$, setting QEWSB $>M_{Z} / 1 \mathrm{GeV}$ in the input results in $M_{S}=$ QEWSB $\mathrm{GeV}$ being fixed.

If INCLUDE_2_LOOP_SCALAR_CORRECTIONS is switched off (0) as in the default case, 2-loop RGEs [31] are used for the Higgs and gaugino masses, $\mu$, Yukawa and gauge couplings but 1-loop RGEs are used for other MSSM parameters. Switching on the 2-loop corrections (1) results in a full 2-loop RGE evolution, but slows the calculation by a factor of approximately three. <numHiggsLoops> may be set to either 2 (default) or 1, and is the number of loops of particles allowed to contribute to the Higgs mass threshold calculation and the tadpoles in the electroweak symmetry breaking conditions.

If softpoint. $\mathrm{x}$ is used without the SLHA interface, default Standard Model inputs are used from the files def.h and lowe.h. The low energy data is encoded in a QedQcd object and must be provided. The default numbers supplied and contained in the QedQcd object are given in units of GeV and running masses are in the $\overline{M S}$ scheme. For the bottom and top masses, either the running mass or the pole mass must be supplied as an input. The type of mass not given for input is calculated by SOFTSUSY at

\footnotetext{
${ }^{5}$ Note that the input value of $\tan \beta$ is the value at $M_{Z}$.
} 
the 3-loop QCD level. We recommend, along with ref. [32], that the running mass be used for $m_{b}$ since there are smaller theoretical errors in the extraction of this quantity from experiment than the pole mass. The scale dependent quantities in this object are then evolved to $M_{Z}$ by the method tomz, to provide the low-scale empirical boundary condition for the rest of the calculation. The MSSM spectrum calculated depends most crucially upon $M_{Z}, \alpha\left(M_{Z}\right), \alpha_{s}\left(M_{Z}\right)$ and input third family fermion masses.

\section{B Sample Program}

We now present the sample program from which it is possible to run SOFTSUSY in a simple fashion. The program we presents here performs a scan in the variable $\tan \beta$, with other parameters as in point CMSSM10.1.1 [33]. It then prints the four pole Higgs masses as a function of $\tan \beta$ in the standard output channel. If there are any problems with the parameter point, the program prints out these instead of the Higgs masses. The most important features of the objects are described in appendix E. The sample program main.cpp has the following form:

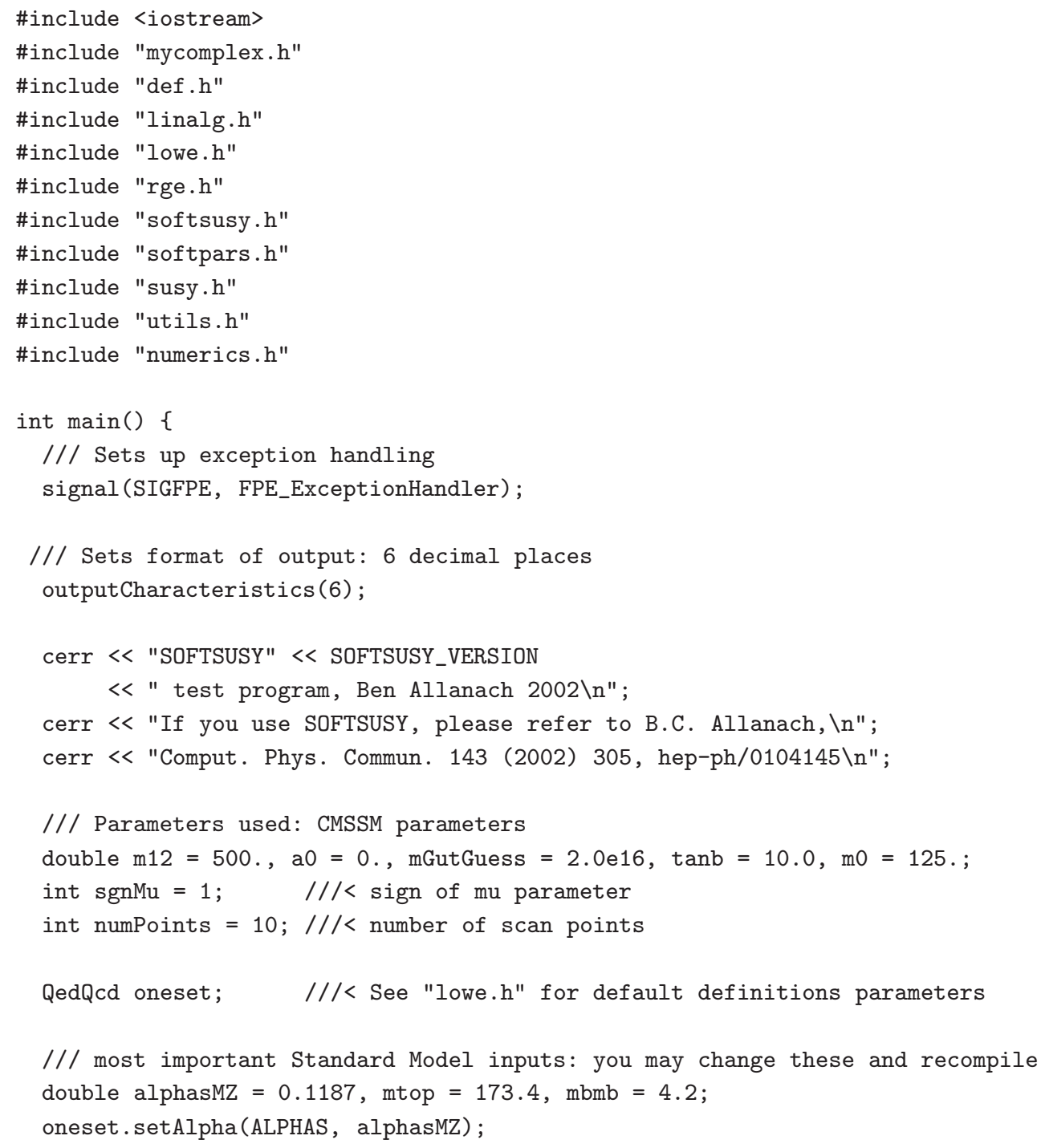




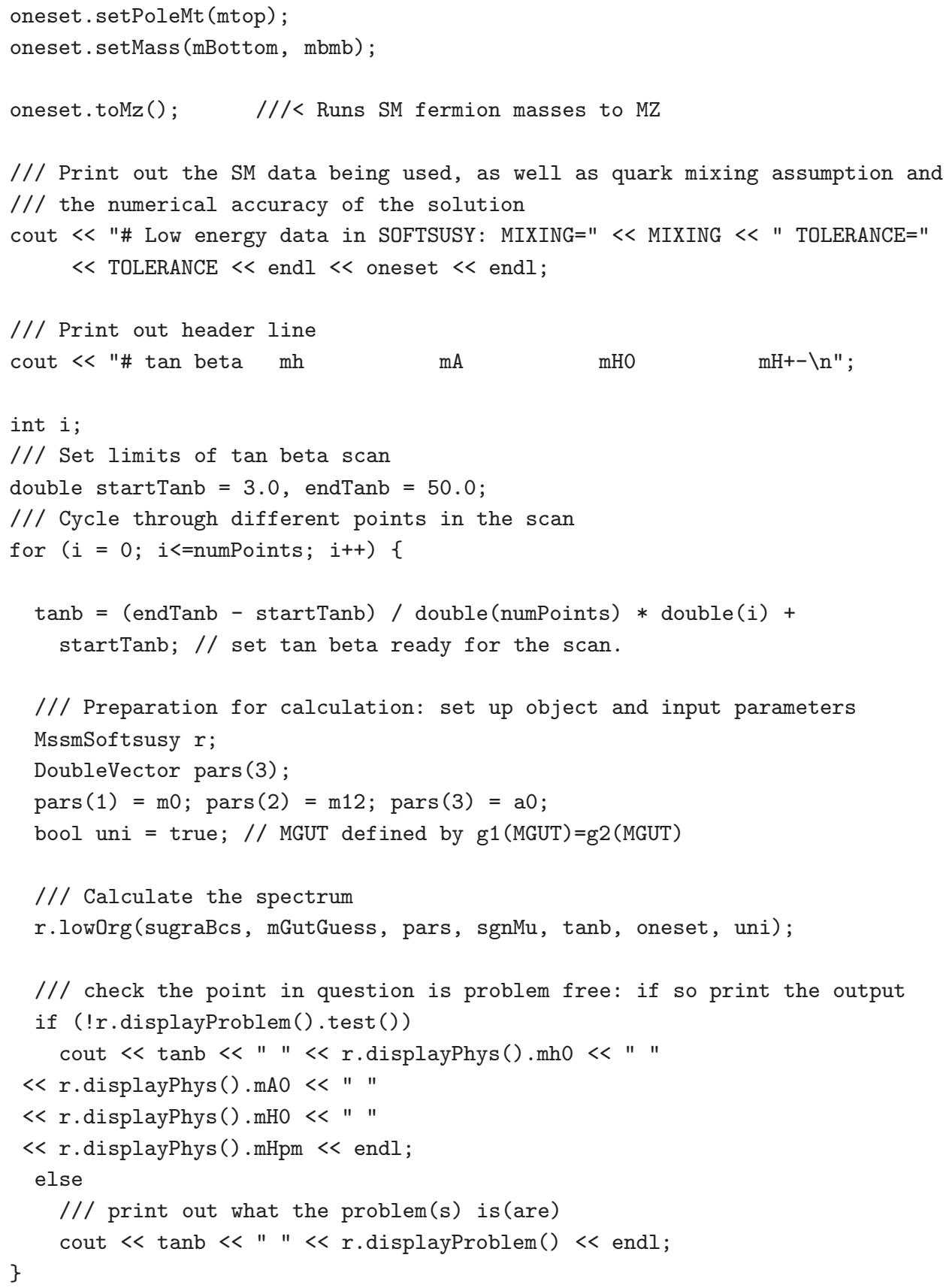

First of all, a function signal is called which attempts to catch any floating point exceptions that may occur during the running of the program. Then, after an initial introductory print-out, the variables specifying the supersymmetry breaking parameters are specified. For these, the same notation as appendix A is used. Next, the important Standard Model inputs are defined and combined with the defaults already present in the QedQcd object. The top running mass is calculated from the pole mass and Standard Model fermion masses and gauge couplings are then run up to $M_{Z}$ with the method toMz. 


\begin{tabular}{ll} 
name & arguments \\
\hline sugraBcs & $m_{0}, m_{1 / 2}, A_{0}$ \\
amsbBcs & $m_{3 / 2}, m_{0}$ \\
gmsbBcs & $n_{5}, m_{m e s s}, \Lambda$
\end{tabular}

Table 2. SUSY breaking boundary conditions available to the user, detailing arguments in order. The asterisk denotes additional information in the text.

If gaugeUnification=true, softsusy will determine mGutGuess from electroweak gauge unification, using the mGutGuess value supplied as an initial guess. The user can supply a void function that sets the supersymmetry breaking parameters from an input DoubleVector. In the sample code given above, this function is sugraBcs and is applied to the MssmSoftsusy object at the scale $M_{G U T}$, which will be determined by the user (but mGutGuess will be used as an initial guess). Other examples of available boundary conditions are given in Table B. The user must supply a DoubleVector containing the numerical values of the arguments, correctly ordered as in Table B. sugraBcs, for example, calls the MssmSoftsusy method standardSugra(m0, $\mathrm{m} 12$, a0), which sets all scalar masses equal to $\mathrm{m} 0$, all gaugino masses to ${ }^{6}-\mathrm{m} 12$ and all trilinear scalar couplings to a0, in the standard universal fashion. If the user desires to write his or her own boundary condition, it must conform to the prototype

void userDefinedBcs (MssmSoftsusy \& $\mathrm{m}$, const DoubleVector \& inputs)

The method low0rg drives the calculation, after which $\tan \beta$ and the Higgs masses are printed out.

One other small extension to the SLHA input is the option of inputting negative SUSY breaking scalar mass squared values as the theoretical boundary condition at the input scale of the theoretical boundary condition. If this is desired, one writes the negative of the mass in the SLHA input file.

\section{Sample Output}

For the recommended SLHA option, the conventions for the output are explained in Ref. [29]. We present the non-SLHA compliant SOFTSUSY output for the main program above, which can be run by the command

\section{/ softsusy.x}

The output obtained was

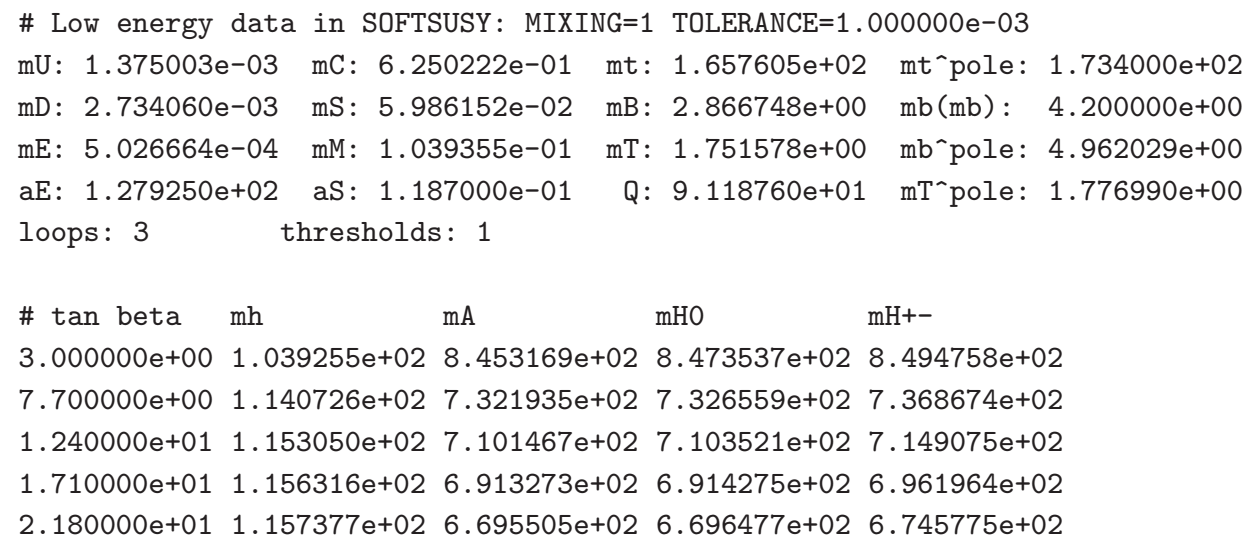

\footnotetext{
${ }^{6}$ With our sign conventions, the CMSSM should impose a common negative gaugino mass.
} 
Firstly, the output details the input parameters, starting with MIXING and TOLERANCE. After the output of the input QedQcd object, various pole Higgs masses are displayed for different values of tan beta, as labelled by the various columns. The columns are, respectively, $\tan \beta, m_{h^{0}}, m_{A^{0}}, m_{H^{0}}$ and $m_{H^{ \pm}}$. In the case that there is a problem with the point, the higgs masses are replaced by a warning: three points have tachyonic staus, and the last has not achieved convergence of the iteration and possesses a negative mass squared for $m_{H^{ \pm}}$. Here, stau tachyon indicates that the stau mass has become imaginary and so the scalar potential minimum does not conserve electromagnetism. For the highest value of $\tan \beta$, No convergence indicates that SOFTSUSY was not able to iterate the calculation to the desired accuracy. hpm tachyon indicates that the charged Higgs masses have become imaginary and the scalar potential minimum does not conserve electromagnetism. The presence of such tachyons rules such parameter points out. We list and explain all of the possible problem flags in the following section.

\section{C.1 Problem flags}

Any associated problems such as negative mass-squared scalars or inconsistent EWSB are flagged at the end of the output. We now list the problems, indicating their meaning:

- If No convergence appears, then SOFTSUSY is indicating that it didn't achieve the accuracy of TOLERANCE within less than 40 iterations. The output of the code is therefore to be considered unreliable and it is not clear from the output whether the point is allowed or disallowed, despite the presence or absence of other warning messages. This error flag often appears near the boundary of electroweak symmetry breaking, (where $\left.\mu\left(M_{S U S Y}\right)=0\right)$ ), where the iterative algorithm is not stable. To calculate the position of the electroweak symmetry boundary, one should interpolate between regions a small distance away from it.

- Non-perturbative indicates that SOFTSUSY encountered couplings reaching Landau poles when evolving, and could not calculate any further. Any results obtained using perturbation theory (for example those of SOFTSUSY) therefore cannot be trusted.

- Infra-red quasi fixed point breached indicates that the parameter point is at a Landau pole of a Yukawa coupling. This should not be a problem provided no other errors are flagged.

- muSqWrongSign indicates that the Higgs minimisation conditions imply that $\mu^{2}<0$, meaning that the desired electroweak minimum is not present in the model. The model is ruled out.

- m3sq indicates that $m_{3}^{2}$ from eq. (3.12) has the incorrect sign, meaning that the desired electroweak minimum is not present in the model. The model is ruled out.

- The tachyon variable labels if a scalar particle other than the Higgs has acquired a negative mass squared, when $M_{Z}^{2}<0$ or when a pole Higgs masses is imaginary. The model is ruled out. 
tachyon is of an enumerated type tachyonType, which relabels the integers $z \in(0,15)$ to indicate which particle is a tachyon according to the rule

$$
\begin{aligned}
& 0=\text { no tachyon, } 1=\tilde{e}, 2=\tilde{\mu}, 3=\tilde{\tau}, 4=\tilde{u}, 5=\tilde{c}, 6=\tilde{c}, 7=\tilde{d}, 8=\tilde{s} \\
& 9=\tilde{b}, 10=h^{0}, 11=A^{0}, 12=H^{ \pm}, 13=\tilde{\nu}_{e}, 14=\tilde{\nu}_{\mu}, 15=\tilde{\nu}_{\tau},
\end{aligned}
$$

respectively.

- noRhoConvergence is flagged when SOFTSUSY cannot calculate the $\rho$ parameter and determine gauge couplings from data, typically because of tachyons or infinities that have crept into the calculation. The other problems are serious enough to rule the model out.

- higgsufb and bProblem indicate that the desired electroweak minimum is in fact a saddle point of the potential, thus the model is ruled out.

- mgutOut0fBounds is flagged if the value of the gauge unification scale predicted by eq. 3.13 is outside the range $10^{4} \mathrm{GeV}<M_{X}<5 \times 10^{17} \mathrm{GeV}$. The GUT-scale has been set to the appropriate limit, and the SOFTSUSY numbers cannot be trusted.

- inaccurateHiggsMass is flagged when the $\overline{D R}$ perturbation series has broken down, and higher order terms are potentially $\sim \mathcal{O}(1)$. This can happen when $\left(M_{3} / m_{\tilde{t}_{1}}\right)^{2}>16 \pi^{2}$, or when $\left(\mu / m_{\tilde{t}_{1}}\right)^{2}>16 \pi^{2}[23]$. The rest of the calculation should be fine, but one cannot trust the higgs masses. One should use some other program that uses the on-shell scheme to calculate Higgs masses for these points ${ }^{7}$.

Thus flags other than No convergence, Infra-red quasi fixed point breached, inaccurateHiggsMass or Non-perturbative indicate an unphysical minimum of the scalar potential, effectively ruling the model point out. Another structure within MssmSoftsusy of type sProblem flags various potential problems with the object, for example the lack of radiative EWSB or negative mass squared scalars (excluding the Higgs mass squared parameters). This structure is shown in table E.7. In addition, the method test prints out if any of the possible data variables flagging problems are true. The higgsufb flag is true if

$$
m_{H_{1}}^{2}+2 \mu^{2}+m_{H_{2}}^{2}-2\left|m_{3}^{2}\right|<0
$$

is not satisfied, implying that the desired electroweak minimum is either a maximum or a saddlepoint of the tree-level Higgs potential [2]. The contents of sPhysical and sProblem can be output with overloaded $\ll$ operators. noConvergence means that the desired accuracy was not reached. nonPerturbative or irqfp flags the existence of a Landau pole in the renormalisation group evolution, and the calculation is not perturbatively reliable so any results should be discarded. All other problems except noConvergence and nonPerturbative should be considered as grounds for ruling the model out. noRhoConvergence occurs when the pseudo-scalar Higgs $A^{0}$ has a negative mass squared (i.e. an invalid electroweak vacuum).

\section{Switches and Constants}

The file def.h contains the switches and constants. If they are changed, the code must be recompiled in order to use the new values. def . cpp contains initial values for global variables (in the SOFTSUSY namespace). These may be changed by the user in their main programs. Table D shows the most important

\footnotetext{
${ }^{7}$ Although we note that there are many different points for which the $\overline{O S}$ scheme has a perturbation series problem and one must use the $\overline{D R}$ instead.
} 


\begin{tabular}{lll} 
variable & default & description \\
\hline ARRAY_BOUNDS_CHECKING & off & Vector and Matrix bounds checking \\
EPSTOL & $10^{-11}$ & Underflow accuracy \\
GMU & $1.1663710^{-5}$ & $G_{\mu}$, Fermi constant from muon decay \\
MZCENT & 91.1876 & Pole mass of the $Z^{0}$ boson $M_{Z}$.
\end{tabular}

Table 3. Switches and constants. Starred entries have more explanation in the text. $G_{\mu}$ is in units of $\mathrm{GeV}^{2}$ and $M_{Z}$ in $\mathrm{GeV}$.

parameters in def.h, detailing the default values that the constants have. $G_{\mu}$ and $M_{Z}$ have been obtained using the latest particle data group numbers [17].

\section{E Object Structure}

We now go on to sketch the objects and their relationship. This is necessary information for generalisation beyond the MSSM. Only methods and data which are deemed important for prospective users are mentioned here, but there are many others within the code itself.

\section{E.1 Linear Algebra}

The SOFTSUSY program comes with its own linear algebra classes: Complex, DoubleVector, DoubleMatrix, ComplexVector, ComplexMatrix. Constructors of the latter four objects involve the dimensions of the object, which start at 1 . Complex objects are constructed with their real and imaginary parts respectively. For example, to define a vector $a_{i=1,2,3}$, a matrix $m_{i=1 \ldots 3, j=1 \ldots 4}$ of type double and a Complex number $b=1-i$ :

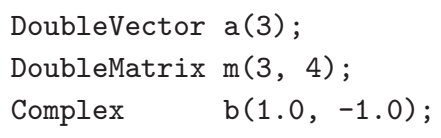

Obvious algebraic operators between these classes (such as multiplication, addition, subtraction) are defined with overloaded operators $*,+,-$ respectively. Elements of the vector and matrix classes are referred to with brackets (). DoubleVector and DoubleMatrix classes are contained within each of the higher level objects that we now describe.

\section{E.2 General Structure}

From a RGE point of view, a particular quantum field theory model consists of a set of couplings and masses defined at some renormalisation scale $\mu$. A set of $\beta$ functions describes the evolution of the parameters and masses to a different scale $\mu^{\prime}$. This concept is embodied in an abstract RGE object, which contains the methods required to run objects of derived classes to different renormalisation scales. The other objects displayed in figure E.2 are particular instances of RGE, and therefore inherit from it. QedQcd objects consist of data on the quark and lepton masses and gauge couplings. They contain the $\beta$ functions for running in an effective QED $\times$ QCD theory below $m_{t}$. An object of class MssmSusy contains the Yukawa couplings, and the three gauge couplings of the MSSM. It also contains the superpotential $\mu$ term (not to be confused with the renormalisation scale), $\tan \beta$, the ratio of the 


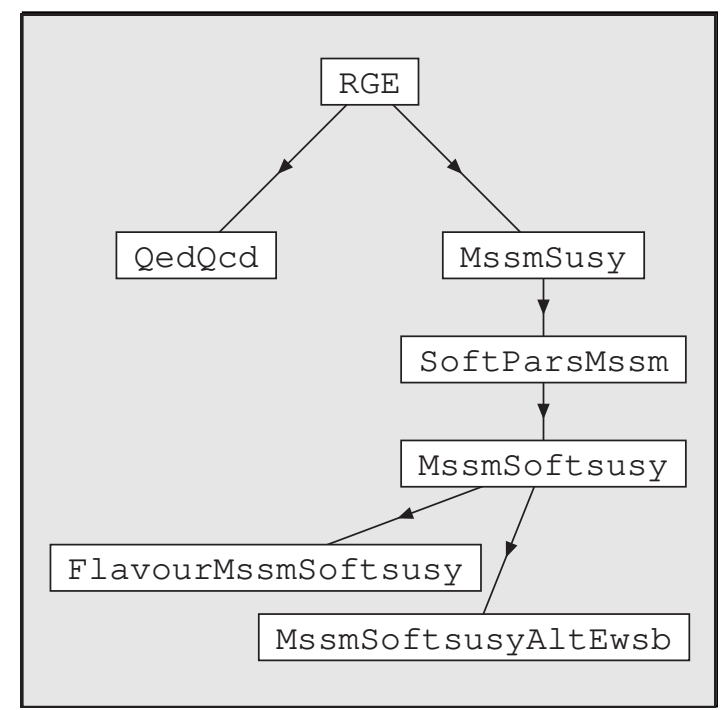

Figure 2. Heuristic high-level object structure of SOFTSUSY. Inheritance is displayed by the lines.

two Higgs doublet VEVs as well as $v=\sqrt{v_{1}^{2}+v_{2}^{2}}$. Its $\beta$ functions are valid in the exact SUSY limit of the MSSM. The major part of the code resides within the MssmSoftsusy class. Objects of this type have all the functionality of MssmSusy, with soft SUSY breaking terms and theoretical boundary conditions contained in the inherited class SoftParsMssm. It also contains an object of type QedQcd which contains weak scale empirical data. Code in the MssmSoftsusy class organises and performs the main part of the calculation. MssmSoftsusyAltEwsb objects are a slight variant of MssmSoftsusy: it takes non-universal Higgs mass boundary conditions at the SUSY breaking scale. FlavourMssmSoftsusy objects are also slight variants: they have full flavour-mixed output and input. In the following, we provide basic information on classes so that users may program using SOFTSUSY. Highly detailed and technical documentation on the program may be obtained from the SOFTSUSY website.

\section{E.3 RGE Class}

The data and important methods in RGE are presented in table E.3. Each of the higher level objects described in this appendix have explicitly named display and set methods that are used to access or change the data contained within each object. In table E.3 (as in the following tables in this section), these accessing methods are listed on the same row as the relevant data variable.

The RGE method runto(mup, eps) will automatically run any derived object to the scale mup with a fractional accuracy of evolution eps. In order to define this evolution, any object that inherits from an RGE must contain three methods: display, set, beta shown in table E.3. DoubleVector display() const must return a vector containing all masses and couplings of the object, in some arbitrary userdefined order. void set(const DoubleVector \& v) must set these couplings given a DoubleVector v defined in the same order as the display function. DoubleVector beta() const must then return the $\beta$ functions in a DoubleVector defined as

$$
\beta_{i}=\frac{d a_{i}}{d \ln \mu}
$$

where $a_{i}$ denotes any mass or coupling of the model. The ordering of the $a_{i}$ must be identical in each of the three methods. 


\begin{tabular}{|c|c|c|}
\hline double $\mathrm{mu}=\mu$ & $\begin{array}{l}\text { renormalisation scale } \\
(\mathrm{GeV})\end{array}$ & $\begin{array}{l}\text { setMu } \\
\text { displayMu }\end{array}$ \\
\hline int numpars & $\begin{array}{l}\text { number of scale dependent } \\
\text { parameters }\end{array}$ & $\begin{array}{l}\text { setPars } \\
\text { howMany }\end{array}$ \\
\hline int loops & accuracy of RGE & $\begin{array}{l}\text { setLoops } \\
\text { displayLoops }\end{array}$ \\
\hline int thresholds & $\begin{array}{l}\text { accuracy level of threshold } \\
\text { computation }\end{array}$ & $\begin{array}{l}\text { setThresholds } \\
\text { displayThresholds }\end{array}$ \\
\hline method & function & \\
\hline $\begin{array}{l}\text { DoubleVector display() } \\
\text { void set(DoubleVector) } \\
\text { DoubleVector beta } \\
\text { runto }\end{array}$ & $\begin{array}{l}\text { displays all running parame } \\
\text { sets all running parameters } \\
\text { displays beta functions of a } \\
\text { runs object to new value of }\end{array}$ & $\begin{array}{l}\text { ers }(*) \\
(*) \\
\text { running parameters }(*) \\
\text { nu }\end{array}$ \\
\hline
\end{tabular}

Table 4. Abstract RGE class. $(*)$ indicates that derived objects must contain these methods (see text).

\begin{tabular}{lll} 
data variable & methods \\
\hline $\begin{array}{l}\text { DoubleVector a } \\
\alpha(\mu), \alpha_{s}(\mu)\end{array}$ & $\overline{M S}$ gauge couplings & $\begin{array}{l}\text { setAlpha } \\
\text { displayAlpha }\end{array}$ \\
\hline $\begin{array}{l}\text { DoubleVector m } \\
m_{f}(\mu)\end{array}$ & $\begin{array}{l}\text { running fermion masses } \\
\text { vector }(1 \ldots 9)(\mathrm{GeV})\end{array}$ & $\begin{array}{l}\text { setMass } \\
\text { displayMass }\end{array}$ \\
\hline $\begin{array}{l}\text { double mtPole, mbPole } \\
\text { double mtauPole }\end{array}$ & pole top/bottom/tau & setPoleMt, setPoleMb \\
$m_{t}^{\text {pole }}, m_{b}^{\text {pole }}, m_{\tau}^{\text {pole }}$ & $(\mathrm{GeV})$ & setPoleMtau \\
& & displayPoleMt displayPoleMb \\
& & displayPoleMtau \\
method & function & \\
\hline runGauge & runs gauge couplings only \\
toMt, toMz & runs fermion masses and gauge couplings \\
& from $Q^{\prime}$ to $m_{t}^{\text {pole }}$ or $M_{Z}$ &
\end{tabular}

Table 5. QedQcd class. $Q^{\prime}$ is defined in the text.

\section{E.4 QedQcd Class}

The QedQcd class contains a DoubleVector of quark and lepton $\overline{M S}$ masses $\left(m_{f}=m_{u, d, e, c, s, \mu, t, b, \tau}(\mu)\right)$, as shown in table E.4. Its contents may be printed to standard output or read from standard input (with the same format in each case) by using the operators $\ll$ or $>$, as can all the non-abstract objects mentioned in this section. The methods $\operatorname{tomz}()$, toMt () act on an initial object defined with each fermion mass $m_{f}$ defined at a scale

$$
Q^{\prime}=\max \left(1 \mathrm{GeV}, m_{f}\left(m_{f}\right)\right)
$$

and gauge couplings at $M_{Z}$. 


\begin{tabular}{|c|c|c|}
\hline data variable & & methods \\
\hline $\begin{array}{l}\text { DoubleMatrix u, d, e } \\
\left(Y_{U}\right)_{i j},\left(Y_{D}\right)_{i j},\left(Y_{E}\right)_{i j}\end{array}$ & $\begin{array}{l}\text { Yukawa couplings } \\
\text { ( } 3 \text { by } 3 \text { matrix })\end{array}$ & $\begin{array}{l}\text { setYukawaElement } \\
\text { setYukawaMatrix } \\
\text { displayYukawaElement } \\
\text { displayYukawaMatrix }\end{array}$ \\
\hline $\begin{array}{l}\text { DoubleVector } \mathrm{g} \\
g_{i}\end{array}$ & $\begin{array}{l}\text { MSSM gauge couplings } \\
(1 \ldots 3) \text { vector }\end{array}$ & $\begin{array}{l}\text { setAllGauge } \\
\text { setGaugeCoupling } \\
\text { displayGauge } \\
\text { displayGaugeCoupling }\end{array}$ \\
\hline $\begin{array}{l}\text { double smu } \\
\mu\end{array}$ & $\begin{array}{l}\text { bilinear Higgs superpotential } \\
\text { parameter }\end{array}$ & $\begin{array}{l}\text { setSusyMu } \\
\text { displaySusyMu }\end{array}$ \\
\hline $\begin{array}{l}\text { double } \operatorname{tanb} \\
\tan \beta\end{array}$ & $\begin{array}{l}\text { ratio of Higgs VEVs (at } \\
\text { current renormalisation scale) }\end{array}$ & $\begin{array}{l}\text { setTanb } \\
\text { displayTanb }\end{array}$ \\
\hline $\begin{array}{l}\text { double hVev } \\
v\end{array}$ & Higgs VEV & $\begin{array}{l}\text { setHvev } \\
\text { displayHvev }\end{array}$ \\
\hline method & \multicolumn{2}{|l|}{ function } \\
\hline setDiagYukawas & \multirow{2}{*}{\multicolumn{2}{|c|}{$\begin{array}{l}\text { calculates and sets all diagonal Yukawa couplings } \\
\text { given fermion masses and a Higgs VEV } \\
\text { calculates quark and lepton masses from Yukawa } \\
\text { couplings }\end{array}$}} \\
\hline getMasses & & \\
\hline $\begin{array}{l}\text { getQuarkMixing } \\
\text { getQuarkMixedYukawas }\end{array}$ & \multicolumn{2}{|c|}{$\begin{array}{l}\text { mixes quark Yukawa couplings from mass to weak basis } \\
\text { sets all entries of quark Yukawa couplings given fermion } \\
\text { masses, Higgs VEV and CKM matrix }\end{array}$} \\
\hline
\end{tabular}

Table 6. MssmSusy class.

\section{E.5 MssmSusy Class}

The operators 《<, > have been overloaded to write or read a MssmSusy object to/from a file stream. Table E.5 shows the data variables and important methods contained in the class. For the Yukawa and gauge couplings, methods exist to either set (or display) one element or a whole matrix or vector of them.

\section{E.6 SoftParsMssm Class}

The operators $\ll<$, $>$ have been overloaded to write or read a softParsMssm object to/from a file stream. Table E.6 shows the data variables and important methods contained in the class. addAmsb() adds anomaly mediated supersymmetry breaking terms [38] to the model's soft parameters. Such terms are proportional to the VEV of a compensator superfield, so $m_{3 / 2}$ in table E. 6 must have been set before addAmsb is used. minimalGmsb (int n5, double lambda, double mMess) applies the messenger scale mMess boundary conditions to the soft masses in minimal gauge-mediated supersymmetry breaking [39]. n5 denotes the number of $5 \oplus \overline{5}$ messenger fields that are present and lambda $(\Lambda)$ is as described in ref. [39]. 


\begin{tabular}{|c|c|c|}
\hline data & & methods \\
\hline $\begin{array}{l}\text { double m32 } \\
m_{3 / 2}\end{array}$ & $\begin{array}{l}\text { compensator } \mathrm{VEV}^{*} \\
(\mathrm{GeV})\end{array}$ & $\begin{array}{l}\text { setM32 } \\
\text { displayGravitino }\end{array}$ \\
\hline $\begin{array}{l}\text { DoubleVector mGaugino } \\
M_{1,2,3}\end{array}$ & $\begin{array}{l}(1 \ldots 3) \text { vector of gaugino } \\
\text { mass parameters }\end{array}$ & $\begin{array}{l}\text { setGauginoMass } \\
\text { displayGaugino }\end{array}$ \\
\hline $\begin{array}{l}\text { DoubleMatrix ua, da, ea } \\
U_{A}, D_{A}, E_{A}\end{array}$ & $\begin{array}{l}\text { (3 by } 3) \text { matrix of trilinear } \\
\text { soft terms }(\mathrm{GeV})\end{array}$ & $\begin{array}{l}\text { setTrilinearElement } \\
\text { displayTrilinearElement } \\
\text { displaySoftA }\end{array}$ \\
\hline $\begin{array}{l}\text { DoubleMatrix mQLsq } \\
\text { mURsq, mDRsq, mLLsq } \\
\text { mSEsq } \\
\left(m_{\tilde{Q}_{L}}^{2}\right),\left(m_{\tilde{u}_{R}}^{2}\right),\left(m_{\tilde{d}_{R}}^{2}\right), \\
\left(m_{\tilde{L}_{L}}^{2}\right),\left(m_{\tilde{e}_{R}}^{2}\right)\end{array}$ & $\begin{array}{l}(3 \text { by } 3) \text { matrices of soft } \\
\text { SUSY breaking masses } \\
\left(\mathrm{GeV}^{2}\right)\end{array}$ & $\begin{array}{l}\text { setSoftMassElement } \\
\text { setSoftMassMatrix } \\
\text { displaySoftMassSquared }\end{array}$ \\
\hline $\begin{array}{l}\text { double m3sq,mH1sq,mH2sq } \\
m_{3}^{2}, m_{H_{1}}^{2}, m_{H_{2}}^{2}\end{array}$ & $\begin{array}{l}\text { Bilinear Higgs parameters } \\
\left(\mathrm{GeV}, \mathrm{GeV}^{2}, \mathrm{GeV}^{2}\right)\end{array}$ & $\begin{array}{l}\text { setM3Squared } \\
\text { setMh1Squared } \\
\text { setMh2Squared } \\
\text { displayM3Squared } \\
\text { displayMh1Squared } \\
\text { displayMh2Squared }\end{array}$ \\
\hline method & function & \\
\hline standardSugra & \multicolumn{2}{|l|}{ Sets all universal soft terms } \\
\hline universalScalars & \multicolumn{2}{|l|}{ Sets universal scalar masses } \\
\hline universalGauginos & \multicolumn{2}{|l|}{ Sets universal gaugino masses } \\
\hline universalTrilinears & \multicolumn{2}{|c|}{ Sets universal soft breaking trilinear couplings } \\
\hline addAmsb & \multicolumn{2}{|c|}{ Adds AMSB soft terms to current object* } \\
\hline minimalGmsb & \multicolumn{2}{|c|}{ Gauge-mediated soft terms used as boundary conditions* } \\
\hline
\end{tabular}

Table 7. SoftParsMssm class data and methods. The asterisk denotes additional information in the text.

\section{E.7 MssmSoftsusy Class}

MssmSoftSusy objects contain a structure sPhysical encapsulating the physical information on the superparticles (pole masses and physical mixings), as shown in table E.7. Another structure of type drBarPars inherits from sPhysical but instead contains information on $\overline{D R}$ masses and mixing angles. MssmSoftSusy objects also contain one of these structures for calculational convenience: the information is used in order to calculate loop corrections to various masses. As table E.7 shows, a method mpzCharginos returns the 2 by 2 complex diagonalisation matrices $U, V$ that result in positive $\overline{D R}$ chargino masses, as defined in ref. [3]. The method mpzNeutralinos is present in order to convert $O$ to the complex matrix $N$ defined in ref. [3] that would produce only positive $\overline{D R}$ neutralino masses. This information, as well as $\overline{D R}$ third family fermion masses are stored in the drBarPars structure.

MssmSoftsusy data variables and accessors can be viewed in table E.7 and the most important

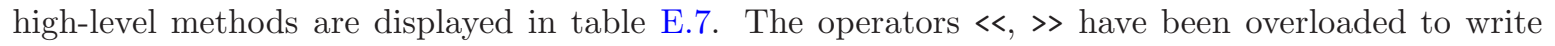
or read MssmSoftusy objects or sPhysical structures to/from a file stream. The driver routine for the 


\begin{tabular}{ll} 
data variable & description \\
\hline double mh0,mA0,mH0,mHpm & $h^{0}, A^{0}, H^{0}, H^{ \pm}$masses \\
DoubleVector msnu & vector of $m_{\tilde{\nu}_{i=1 \ldots 3}}$ masses \\
DoubleVector mch,mneut & vectors of $m_{\chi^{ \pm}}, m_{\chi_{1=1} \ldots 2}{ }_{i=1 \ldots 4}$ respectively \\
double mGluino & gluino mass $m_{\tilde{g}}$ \\
DoubleMatrix mixNeut & 4 by 4 orthogonal neutralino mixing matrix $O$ \\
double thetaL, thetaR & $\theta_{L, R}$ chargino mixing angles \\
double thetat, thetab & $\theta_{t, b}$ sparticle mixing angles \\
double thetatau, thetaH & $\theta_{\tau}, \alpha$ sparticle and Higgs mixing angles \\
DoubleMatrix mu, md, me & $(2$ by 3$)$ matrices of up squark, down squark and \\
& charged slepton masses \\
double t10V1Ms, t20V2Ms & 2-loop tadpoles $t_{1} / v_{1}$ and $t_{2} / v_{2}$ evaluated at $M_{S}$ \\
double t10V1Ms1loop, t20V2Ms1loop & 1-loop tadpoles $t_{1} / v_{1}$ and $t_{2} / v_{2}$ evaluated at $M_{S}$
\end{tabular}

Table 8. sPhysical structure. Masses are pole masses, and stored in units of GeV. Mixing angles are in radian units.

\begin{tabular}{ll} 
data variable & description \\
\hline $\begin{array}{l}\text { double } \mathrm{mt}, \mathrm{mb}, \mathrm{mtau} \\
m_{t}(Q), m_{b}(Q), m_{\tau}(Q)\end{array}$ & Third family fermion masses \\
\hline DoubleVector mnBpmz, mchBpmz & Absolute neutralino and chargino masses \\
$m_{\chi_{i}^{0}}, m_{\chi_{i}^{ \pm}}$ & $(1 \ldots 4,1 \ldots 2)$ vectors \\
\hline ComplexMatrix nBpmz & Neutralino mixing matrix \\
$N$ & $(4$ by 4 complex matrix $)$ \\
\hline ComplexMatrix uBpmz, vBpmz & Chargino mixing matrices \\
$U, V$ & $(2$ by 2 complex matrices $)$ \\
\hline & \\
name & function \\
\hline mpzNeutralino & Gives mixing matrices required to make \\
mpzChargino & neutralino masses positive* \\
& Gives mixing matrices required to make \\
& chargino masses positive*
\end{tabular}

Table 9. drBarPars structure. Masses are in the $\overline{D R}$ scheme, and stored in units of GeV. Mixing angles are in radian units. Functions marked with an asterisk are mentioned in the text.

RGE evolution and unification calculation is

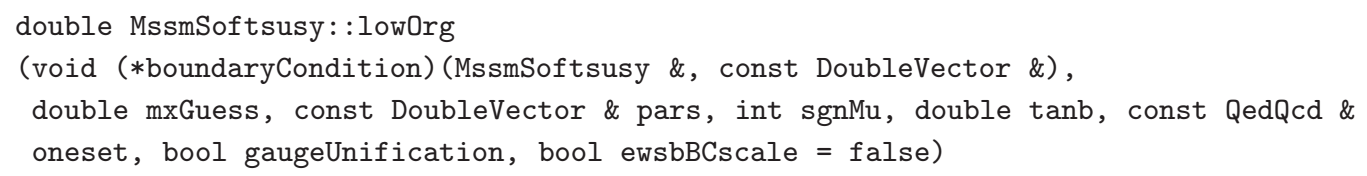

The user-supplied boundaryCondition function sets the soft parameters according to the elements of the supplied DoubleVector at mxGuess, as discussed in appendix B. If gaugeUnification is true, the scale that unifies the electroweak gauge couplings is used and returned by the function. If gaugeUnification is 


\begin{tabular}{ll} 
data variable & flags \\
\hline mgutOutOfBounds & $M_{X}>5 \times 10^{17} \mathrm{GeV}$ or $M_{X}<10^{4} \mathrm{GeV}$ \\
irqfp & in a region with a Landau pole \\
noRhoConvergence & the $\rho$ iterative routine doesn't converge \\
noConvergence & the main iteration routine doesn't converge \\
tachyon & a non-Higgs scalar has negative mass squared \\
muSqWrongSign & $\mu^{2}$ from eq. (3.11) negative \\
m3sq & $m_{3}^{2}$ from eq. (3.12) has incorrect sign \\
higgsUfb & eq. (C.1) is not satisfied \\
nonperturbative & a Landau pole was reached below the unification scale \\
noMuConvergence & $\mu$ could not be calculated reliably \\
inaccurateHiggsMass & Higgs masses cannot be trusted \\
problemThrown & numerical exception occurred during run (infinities etc)
\end{tabular}

Table 10. sProblem structure. All data variables are boolean values except for tachyon, which is of type tachyonType. See Section C.1 for more details.

\begin{tabular}{lll} 
data & & methods \\
\hline double mwPred & pole $M_{W}$ prediction & setMw \\
$M_{W}$ & $(\mathrm{GeV})$ & displayMw \\
\hline double msusy & Minimisation scale & displayMsusy \\
$M_{S}$ & $(\mathrm{GeV})$ & setMsusy \\
\hline QedQcd dataset & $M_{Z}$ boundary condition on & setData \\
& Standard Model couplings & displayDataSet \\
\hline sProblem problem & problem flags & displayProblem \\
& & flagIrqfp, flagB \\
& & flagNonperturbative \\
& & flagTachyon, flagHiggsufb \\
& & flagNoConvergence \\
& & flagNoMuConvergence \\
& & flagNoRhoConvergence \\
& & flagMusqwrongsign \\
& & flagAllProblems \\
\hline DrBarPars forLoops & $\overline{D R}$ masses and mixings & displayDrBarPars \\
& & setDrBarPars \\
\hline sPhysical physpars & pole masses and mixings & displayPhys \\
& & setPhys
\end{tabular}

Table 11. MssmSoftsusy class data and accessor methods.

false, the function simply returns mxGuess. ewsbBCscale is an optional argument: if one wishes to impose the soft SUSY breaking boundary conditions at $M_{S U S Y}$ rather than $M_{X}$, one should call lowOrg with the value true for this argument. Omitting the argument, or giving it the default false value means that $M_{X}$ will be used instead. pars contains a DoubleVector of soft SUSY breaking 


\begin{tabular}{ll} 
name & function \\
\hline lowOrg & Driver routine for whole calculation* \\
methodBoundaryCondition & Boundary condition for derived objects \\
itLowsoft & Performs the iteration between $M_{Z}$ and \\
& unification scale \\
sparticleThresholdCorrections & $\overline{D R}$ radiative corrections to Standard Model \\
& couplings at $M_{Z}$ \\
physical & Calculates sparticle pole masses and mixings \\
calcDrBarPars & Calculates $\overline{D R}$ pole masses and mixings \\
rewsb & Sets $\mu, B$ from EWSB conditions \\
fineTune & Calculates fine-tuning for soft parameters* \\
getVev & and $h_{t}$ \\
& Calculates VEV $v^{D R}$ at current scale from $Z$ \\
calcSinthdrbar & self-energy and gauge couplings \\
& Calculates $s_{W}^{D R}$ at current scale from \\
calcMs & gauge couplings \\
printShort & Calculates $M_{S}$ \\
printLong & short list of important parameters printed out \\
outputFcncs & to standard output in columns \\
lesHouchesAccordOutput & long list of important parameters printed out \\
& to standard output in columns \\
& prints a list of flavour-changing $\delta$ parameters* \\
& prints output in SLHA [29] format \\
& calculates then returns sin ${ }^{l} \theta_{\text {eff }}$ \\
&
\end{tabular}

Table 12. MssmSoftsusy methods and related functions. Functions marked with an asterisk are mentioned in the text.

parameters to be applied as the theoretical boundary condition. sgnMu is the sign of the superpotential $\mu$ parameter, tanb is the value of $\tan \beta\left(M_{Z}\right)$ required and oneset contains the $M_{Z}$ scale low energy data.

The fine tuning (as defined in sec. 3) can be calculated with the method

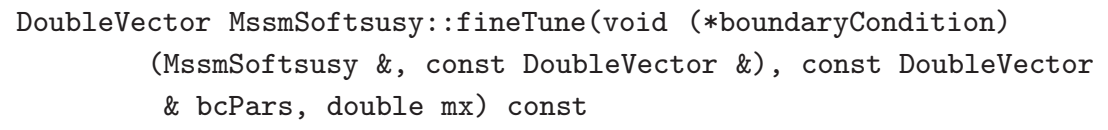

This function should only be applied to an MssmSoftsusy object which has been processed by low0rg. $\mathrm{mx}$ is the unification scale and boundaryCondition is the function that sets the unification scale soft parameters, as discussed above. In derived objects, the virtual method methodBoundaryCondition may be used to set data additional to MssmSoftsusy from the boundaryCondition function. The method outputs the fine-tuning of a parameter $a_{i=1 \ldots n}$ in the bcPars $(n+3)$ DoubleVector, with the $(n+1, n+$ $2, n+3)^{t h}$ element of bcPars being the fine-tuning with respect to the Higgs potential parameters $(\mu$ and $B$ ) and the top Yukawa coupling $\left(h_{t}\right)$ respectively. fineTune is an optional feature. sinSqThetaEff() returns a double number corresponding to a full one-loop calculation of the quantity $\sin ^{2} \theta_{\text {eff }}^{l}$. It does not contain any 2-loop corrections, and may not be accurate enough for precision electroweak fits. 


\section{E.8 MssmSoftsusyAltEwsb Class}

The MssmSoftsusyAltEwsb class, which inherits directly from MssmSoftsusy, adds two private data variables: muCond and mAcond, both massive parameters in units of GeV. They fix the boundary conditions on $\mu\left(M_{S U S Y}\right)$ and the pole pseudo-scalar Higgs mass $m_{A}$ (pole). In this case, $m_{A}\left(M_{S U S Y}\right)$ is extracted from the input $m_{A}$ (pole) via $m_{A}^{2}\left(M_{S U S Y}\right)=m_{A}^{2}$ (pole $)+\Pi_{A A}\left(M_{S U S Y}\right)$, where $\Pi_{A A}\left(M_{S U S Y}\right)$ is the MSSM pseudo-scalar self-energy correction. The Higgs mass squared parameters $m_{H_{1,2}}^{2}$ are not set at $M_{G U T}$ : they are instead set at $M_{S U S Y}$ by solving the simultaneous equations $3.11,3.12$ and using the relationship between $m_{3}^{2}\left(M_{S U S Y}\right)$ and $m_{A}\left(M_{S U S Y}\right)$ :

$$
\begin{aligned}
& m_{H_{1}}^{2}=\sin ^{2} \beta\left(m_{A}^{2}+M_{Z}^{2}\right)+\frac{t_{1}}{v_{1}}\left(1-\sin ^{4} \beta\right)-\sin ^{2} \beta \cos ^{2} \beta \frac{t_{2}}{v_{2}}-\left(\mu^{2}+\frac{1}{2} M_{Z}^{2}\right) \\
& m_{H_{2}}^{2}=\cos ^{2} \beta\left(m_{A}^{2}+M_{Z}^{2}\right)+\frac{t_{2}}{v_{2}}\left(1-\cos ^{4} \beta\right)-\sin ^{2} \beta \cos ^{2} \beta \frac{t_{1}}{v_{1}}-\left(\mu^{2}+\frac{1}{2} M_{Z}^{2}\right),
\end{aligned}
$$

where all quantities in eq. E.3 are running parameters evaluated at $M_{S U S Y}$. This option is covered under the SLHA input parameters EXTPAR 23,26 [29].

\section{E.9 FlavourMssmSoftsusy Class}

FlavourMssmSoftsusy objects inherit directly from MssmSoftsusy with the addition of the variables shown in Table E.9. Some of these constitute the angles of the PMNS and CKM matrices in the standard parameterisation of eq. 3.7. The lesHouchesAccordOutput method has been overloaded to provide flavour violating input and output in accordance with the SLHA2 conventions [40]. Note that SOFTSUSY currently does not contain CP-violating complex phases, despite the inclusion of $\delta$. Thus, when CKM angles are input via the SLHA2 in the Wolfenstein parameterisation, the magnitude of the 13 entry is fit to $\sin \theta_{13}$ as in eq. 3.8 and $\delta$ is set to zero (for now). The relevant method is

void FlavourMssmSoftsusy: :setAngles

(double lambda, double aCkm, double rhobar, double etabar)

Currently, an identical parameterisation is used for the PMNS matrix that describes lepton mixing, except $\theta_{i j}$ is replaced by $\bar{\theta}_{i j}$. There is currently no provision for a CP-violating phase. Effective light neutrino masses are also included in the object.

An optional feature intended for studies of flavour-changing neutral currents (FCNCs) is the method

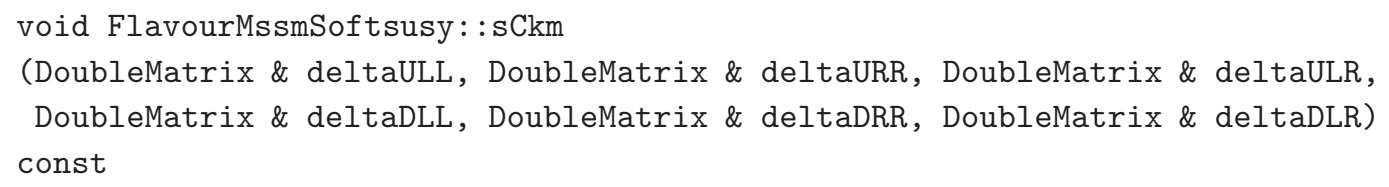

which calculates the parameters $\left(\delta_{L L, L R, R R}^{u, d}\right)_{i j}$ calculated in the mass-squared-insertion approximation (after a rotation to the super CKM basis), as defined in Ref. [10].

The relationship between the super CKM basis of the mass matrices (including one-loop corrections) and the pole-mass basis as described by SLHA2, is contained in the structure flavourPhysical. We list the relevant data in Table E.9. Note that, in the case that CMSSM type inputs are listed in the SLHA2 as well as the flavour input parameters $\hat{T}_{U, D, E}$ (assumed to be in the superCKM basis), a special procedure is required to implement both types of term. At $M_{X}$, the $A_{0}$ terms are added in the interaction basis. There is then a transformation to the super CKM basis, where any values of $\hat{T}_{U, D, E}$ input over-write the trilinear terms. Finally, the trilinear terms are transformed back to the interaction basis. 


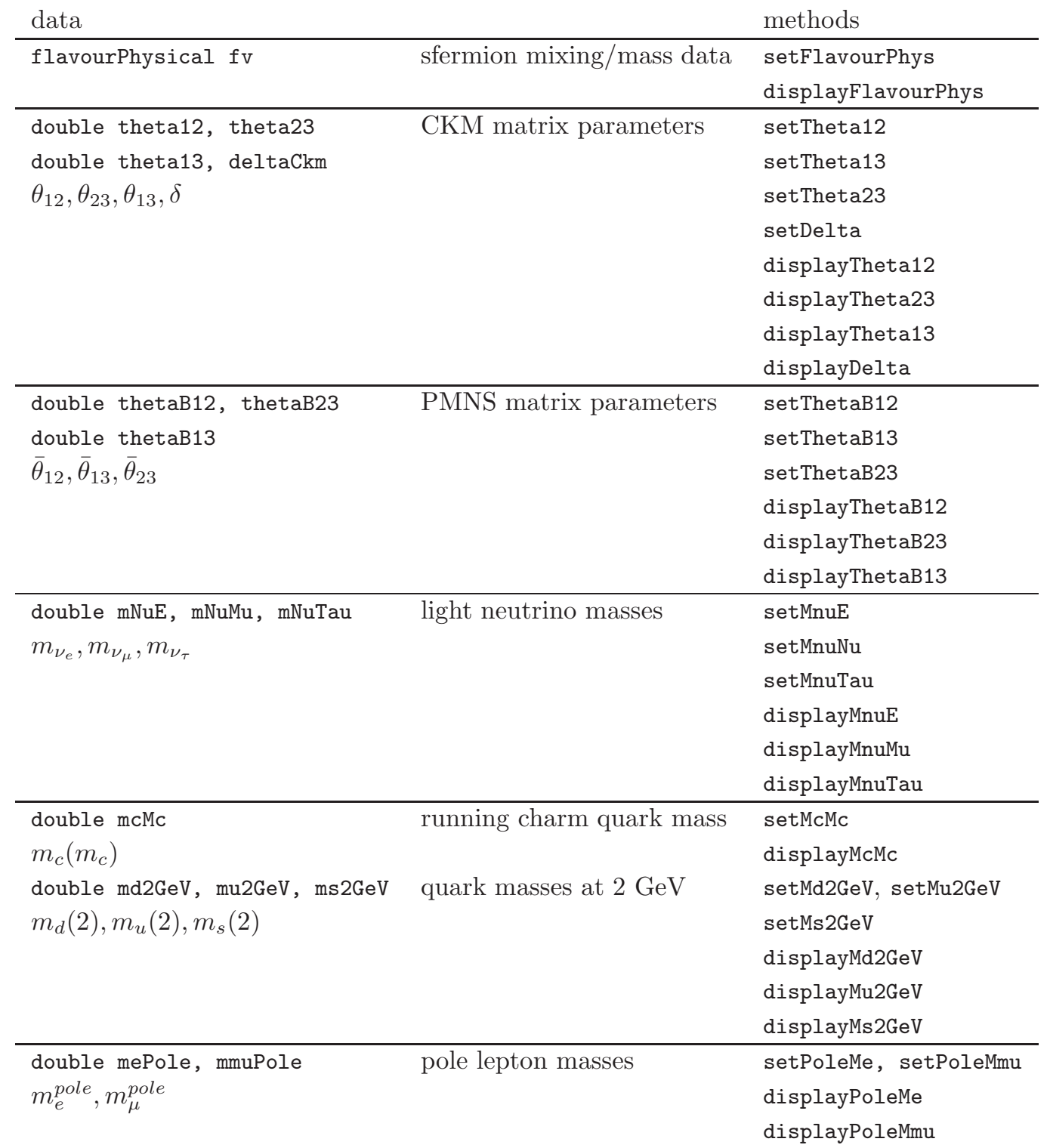

Table 13. FlavourMssmSoftsusy class data and accessor methods. All angles are measured in radians and masses are measured in GeV.

\section{Acknowledgments}

This work has been partially supported by PPARC, STFC and the Aspen Center for Physics. We would like to thank A. Djouadi, S. Kraml and W. Porod for help with detailed comparisons [9] with the codes SUSPECT, ISA JET and SPHEno, S. Akula, P. Athron, M. Baak, M. Badziak, G. Bélanger, F. Borzumati, F. Boudjema, F. Brummer, M. Eads, M. Hamer, J. Hetherington, M. Ibe, J. Kersten, S. Kom, S. Krippendorf, S. Kulkarni, K. Ishikawa, R. Lu, S. Martin, P. Meade, N. Mahmoudi, A. Pukhov, M. Ramage, R. Ruiz, S. Sekmen, A. Sheplyakov, P. Slavich, P. Skands, J. Smidt, C. Tamarit, S. Tet- 


\begin{tabular}{ll} 
data & description \\
\hline DoubleMatrix dSqMix & $6 \times 6$ down squark mixing \\
DoubleMatrix uSqMix & $6 \times 6$ up squark mixing \\
DoubleVector msD & 6 mass-ordered down squark masses \\
DoubleVector msU & 6 mass-ordered up squark masses \\
DoubleMatrix eSqMix & $6 \times 6$ charged slepton mixing matrix \\
DoubleMatrix nuSqMix & $3 \times 3$ sneutrino mixing matrix \\
DoubleVector msE & 6 mass-ordered charged slepton masses \\
DoubleVector msNu & 3 mass-ordered sneutrino masses
\end{tabular}

Table 14. flavourPhysical structure. All mass parameters are pole masses, stored in units of GeV. The mixing matrix definitions exactly coincide with those in the SLHA2 [40] and describe the transformation between the loop-corrected super CKM basis and the mass basis.

suo, T. Watari and A. Wingerter for bug finding and coding suggestions, I. Gafton, J. Holt, F. Krauss, D. Sanford and F. Yu for suggestions on the draft, D. Ross for information on the Passarino-Veltman integrals and K. Matchev for other useful discussions.

\section{References}

[1] G. G. Ross and R. G. Roberts, Minimal supersymmetric unification predictions, Nucl. Phys. B377 (1992) 571-592.

[2] V. Barger, M. S. Berger, and P. Ohmann, The supersymmetric particle spectrum, Phys. Rev. D49 (1994) 4908-4930, [hep-ph/9311269].

[3] D. M. Pierce, J. A. Bagger, K. Matchev, and R. jie Zhang, Precision corrections in the minimal supersymmetric standard model, Nucl. Phys. B491 (1997) 3-67, [hep-ph/9606211].

[4] MSSM Working Group Collaboration, J. K. A. Djouadi and G. Moultaka, Suspect: a program for the supersymmetric spectrum, hep-ph/9901246.

[5] H. Baer, F. E. Paige, S. D. Protopopescu, and X. Tata, Isajet 7.48: A monte carlo event generator for $p$ $p$, anti-p $p$, and $e+e$-reactions, hep-ph/0001086.

[6] B. C. Allanach, J. P. J. Hetherington, M. A. Parker, and B. R. Webber, Naturalness reach of the large hadron collider in minimal supergravity, JHEP 08 (2000) 017, [hep-ph/0005186].

[7] G. Corcella et. al., Herwig 6: An event generator for hadron emission reactions with interfering gluons (including supersymmetric processes), JHEP 01 (2001) 010, see the

HERWIG web page http://hepwww.rl.ac.uk/theory/seymour/herwig/, [hep-ph/0011363].

[8] B. C. Allanach, SUSY Predictions and SUSY Tools at the LHC, Eur. Phys. J. 59 (2009) 427, arXiv:0805.2088 [hep-ph].

[9] B. C. Allanach, Theoretical Uncertainties in Sparticle Mass Predictions, Snowmass Proceedings 2001, [hep-ph/0110227]; B. C. Allanach et al. The Beyond the Standard Model Working Group: Summary Report, Les Houches proceedings 2002, [hep-ph/0204031]. B. C. Allanach, S. Kraml and W. Porod, Comparison of SUSY mass spectrum calculations, SUSY 2002 proceedings, [hep-ph/0207314]; B. C. Allanach, S. Kraml and W. Porod, Theoretical uncertainties in sparticle mass predictions from computational tools, JHEP 0303 (2003) 016, hep-ph/0302102; B. C. Allanach, G. Bélanger, F. Boudjema, A. Pukhov and W. Porod, Uncertainties in Relic Density Calculations in mSUGRA, 
hep-ph/0402161; ibid in Beyond the Standard Model Working Group: Summary Report, Les Houches "Physics at TeV Colliders 2003", hep-ph/0402295.

[10] F. Gabbiani, E. Gabrielli, A. Masiero, and L. Silvestrini, A complete analysis of fcnc and cp constraints in general susy extensions of the standard model, Nucl. Phys. B477 (1996) 321-352, [hep-ph/9604387].

[11] S. G. Gorishnii, A. L. Kataev, S. A. Larin, and L. R. Surguladze, Corrected three loop qcd correction to the correlator of the quark scalar currents and gamma (tot) (h0 $\rightarrow$ hadrons), Mod. Phys. Lett. A5 (1990) 2703-2712.

[12] O. V. Tarasov, A. A. Vladimirov, and A. Y. Zharkov, The gell-mann-low function of qcd in the three loop approximation, Phys. Lett. B93 (1980) 429-432.

[13] S. G. Gorishnii, A. L. Kataev, and S. A. Larin, Next next-to-leading perturbative qcd corrections and light quark masses, Phys. Lett. B135 (1984) 457.

[14] K. G. Chetyrkin, B. A. Kniehl, and M. Steinhauser, Strong coupling constant with flavour thresholds at four loops in the ms-bar scheme, Phys. Rev. Lett. 79 (1997) 2184-2187, [hep-ph/9706430].

[15] L. V. Avdeev and M. Y. Kalmykov, Pole masses of quarks in dimensional reduction, Nucl. Phys. B502 (1997) 419, [hep-ph/9701308].

[16] A. Bednyakov, A. Onishchenko, V. Velizhanin and O. Veretin, Two-loop O( $\left.\alpha_{s}^{2}\right)$ MSSM corrections to the pole masses of heavy quarks, [hep-ph/0210258];

[17] Particle Data Group Collaboration, see the latest Review of Particle Physics on [http://pdg.lbl.gov/] D. E. Groom et. al., Review of particle physics, Eur. Phys. J. C15 (2000) 1.

[18] J. A. Casas, J. R. Espinosa, and H. E. Haber, The higgs mass in the mssm infrared fixed point scenario, Nucl. Phys. B526 (1998) 3-20, [hep-ph/9801365].

[19] S. P. Martin, Two-loop effective potential for a general renormalizable theory and softly broken supersymmetry, Phys. Rev. D65 (2002) 116003 [hep-ph/0111209].

[20] Y. Yamada, Two-loop renormalization of tan(beta) and its gauge dependence, Phys. Lett. B530 (2002) $174[$ hep-ph/0112251].

[21] A. Dedes and P. Slavich, Two-loop corrections to radiative electroweak symmetry breaking in the mssm, hep-ph/0212132.

[22] A. Katsikatsou, A. B. Lahanas, D. V. Nanopoulos, and V. C. Spanos, On the radiative corrections to the pseudo-scalar higgs boson mass, Phys. Lett. B501 (2001) 69-77, [hep-ph/0011370].

[23] G. Degrassi, P. Slavich, and F. Zwirner, On the neutral higgs boson masses in the mssm for arbitrary stop mixing, Nucl. Phys. B611 (2001) 403-422, [hep-ph/0105096].

[24] A. Brignole, G. Degrassi, P. Slavich, and F. Zwirner, On the o(alpha**2(t)) two-loop corrections to the neutral higgs boson masses in the mssm, Nucl. Phys. B631 (2002) 195-218, [hep-ph/0112177].

[25] A. Brignole, G. Degrassi, P. Slavich, and F. Zwirner, On the two-loop sbottom corrections to the neutral higgs boson masses in the mssm, Nucl. Phys. B643 (2002) 79-92, [hep-ph/0206101].

[26] A. Dedes, G. Degrassi and P. Slavich, On the two-loop Yukawa corrections to the MSSM Higgs boson masses at large tan(beta), hep-ph/0305127.

[27] R. Barbieri and A. Strumia, About the fine-tuning price of lep, Phys. Lett. B433 (1998) 63-66,

[28] B. de Carlos and J. A. Casas, One loop analysis of the electroweak breaking in supersymmetric models and the fine tuning problem, Phys. Lett. B309 (1993) 320-328, [hep-ph/9303291].

[29] P. Skands et al, Interfacing SUSY Spectrum Calculators, Decay Packages and Event Generators, JHEP 0407 (2004) 036, [hep-ph/0311123]. 
[30] B. C. Allanach, A. Djouadi, J. L. Kneur, W. Porod and P. Slavich, Precise determination of the neutral Higgs boson masses in the MSSM arXiv:hep-ph/0406166; B. C. Allanach, G. A. Blair, S. Kraml, H. U. Martyn, G. Polesello, W. Porod and P. M. Zerwas, Reconstructing supersymmetric theories by coherent LHC / LC analyses, arXiv:hep-ph/0406166.

[31] S. P. Martin and M. T. Vaughn, Two Loop Renormalization Group Equations For Soft Supersymmetry Breaking Couplings, Phys. Rev. D 50 (1994) 2282 [arXiv:hep-ph/9311340].

[32] H. Baer, J. Ferrandis, K. Melnikov and X. Tata, Relating bottom quark mass in $\overline{D R}$ and $\overline{M S}$ schemes, Phys. Rev. D66 (2002) 074007, [hep-ph/0207126].

[33] S. S. AbdusSalam, B. C. Allanach, H. K. Dreiner, J. Ellis, U. Ellwanger, J. Gunion, S. Heinemeyer and M. Kraemer et al., Benchmark Models, Planes, Lines and Points for Future SUSY Searches at the LHC, Eur. Phys. J. C 71 (2011) 1835 [arXiv:1109.3859 [hep-ph]].

[34] B.C. Allanach et al, The Snowmass Points and Slopes: Benchmarks for SUSY Searches, Eur. Phys. J. C25 (2002) 113, [hep-ph/0202233].

[35] M. Muhlleitner, A. Djouadi and Y. Mambrini, SDECAY: A Fortran code for the decays of the supersymmetric particles in the MSSM [hep-ph/0311167].

[36] T. Sjostrand, L. Lonnblad, S. Mrenna and P. Skands, PYTHIA 6.3 physics and manual, [hep-ph/0308153].

[37] G. Belanger, F. Boudjema, A. Pukhov and A. Semikov, micrOMEGAs: A program for calculating the relic density in the MSSM, Comput. Phys. Comm. 149 (2002) 103, [hep-ph/0112278].

[38] L. Randall and R. Sundrum, Out of this world supersymmetry breaking, Nucl. Phys. B557 (1999) 79, [hep-th/9810155].

[39] S. Ambrosanio, G. D. Kribs and S. P. Martin, Signals for gauge-mediated supersymmetry breaking models at the CERN LEP2 collider, Phys. Rev. D56 (1997) 1761 [hep-ph/9703211].

[40] B.C. Allanach et al, SUSY Les Houches Accord 2, arXiv:0801.0045 [hep-ph]. 